\title{
Article \\ Phytochemical Constituents and Allelopathic Potential of Parthenium hysterophorus L. in Comparison to Commercial Herbicides to Control Weeds
}

\author{
Mst. Motmainna ${ }^{1}$, Abdul Shukor Juraimi ${ }^{1, *}$, Md. Kamal Uddin ${ }^{2}$, Norhayu Binti Asib ${ }^{3} \mathbb{D}_{\text {, }}$ \\ A. K. M. Mominul Islam ${ }^{4}\left(\mathbb{D}\right.$, Muhammad Saiful Ahmad-Hamdani ${ }^{1}$ (D) and Mahmudul Hasan ${ }^{1}$ (D) \\ 1 Department of Crop Science, Faculty of Agriculture, Universiti Putra Malaysia, \\ Serdang 43400, Selangor, Malaysia; gs51794@student.upm.edu.my (M.M.); \\ s_ahmad@upm.edu.my (M.S.A.-H.); gs53801@student.upm.edu.my (M.H.) \\ 2 Department of Land Management, Faculty of Agriculture, Universiti Putra Malaysia, \\ Serdang 43400, Selangor, Malaysia; mkuddin@upm.edu.my \\ 3 Department of Plant Protection, Faculty of Agriculture, Universiti Putra Malaysia, \\ Serdang 43400, Selangor, Malaysia; norhayuasib@upm.edu.my \\ 4 Department of Agronomy, Faculty of Agriculture, Bangladesh Agricultural University, \\ Mymensingh 2202, Bangladesh; akmmominulislam@bau.edu.bd \\ * Correspondence: ashukur@upm.edu.my
}

Citation: Motmainna, M.; Juraimi, A.S.; Uddin, M.K.; Asib, N.B.; Islam, A.K.M.M.; Ahmad-Hamdani, M.S.;

Hasan, M. Phytochemical

Constituents and Allelopathic

Potential of Parthenium hysterophorus L. in Comparison to Commercial Herbicides to Control Weeds. Plants 2021, 10, 1445. https://doi.org/ 10.3390/plants10071445

Academic Editors: Alessia Restuccia and Aurelio Scavo

Received: 17 May 2021

Accepted: 11 June 2021

Published: 15 July 2021

Publisher's Note: MDPI stays neutral with regard to jurisdictional claims in published maps and institutional affiliations.

Copyright: (c) 2021 by the authors. Licensee MDPI, Basel, Switzerland. This article is an open access article distributed under the terms and conditions of the Creative Commons Attribution (CC BY) license (https:// creativecommons.org/licenses/by/ $4.0 /)$.

\begin{abstract}
The allelopathic effect of various concentrations $\left(0,6.25,12.5,50\right.$ and $\left.100 \mathrm{~g} \mathrm{~L}^{-1}\right)$ of Parthenium hysterophorus methanol extract on Cyperus iria was investigated under laboratory and glasshouse conditions. No seed germination was recorded in the laboratory when P. hysterophorus extract was applied at $50 \mathrm{~g} \mathrm{~L}^{-1}$. In the glasshouse, C. iria was mostly injured by P. hysterophorus extract at $100 \mathrm{~g} \mathrm{~L}^{-1}$. The phytochemical constituents of the methanol extract of P. hysterophorus were analyzed by LC-ESI-QTOF-MS=MS. The results indicated the presence of phenolic compounds, terpenoids, alkaloids, amino acids, fatty acids, piperazines, benzofuran, indole, amines, azoles, sulfonic acid and other unknown compounds in P. hysterophorus methanol extract. A comparative study was also conducted between $P$. hysterophorus extract $\left(20,40\right.$ and $\left.80 \mathrm{~g} \mathrm{~L}^{-1}\right)$ with a synthetic herbicide (glyphosate and glufosinate ammonium at $2 \mathrm{~L} \mathrm{ha}^{-1}$ ) as a positive control and no treatment (negative control) on Ageratum conyzoides, Oryza sativa and C. iria. The growth and biomass of test weeds were remarkably inhibited by $P$. hysterophorus extract. Nevertheless, no significant difference was obtained when $P$. hysterophorus extract $\left(80 \mathrm{~g} \mathrm{~L}^{-1}\right)$ and synthetic herbicides (glyphosate and glufosinate ammonium) were applied on $A$. conyzoides.
\end{abstract}

Keywords: allelopathy; phytochemicals; P. hysterophorus; germination; growth

\section{Introduction}

Cyperus iria L. (family: Cyperaceae) is a smooth, tufted sedge weed of lowland rice worldwide and is also a common weed in upland fields of 22 countries [1]. This weed is also reported to appear in dry, direct-seeded rice fields in 21 countries and wet-seeded rice in 11 countries [2]. The roots of $C$. iria are numerous, yellowish-red, short and fibrous. The leaves are usually shorter than culm, $1-8 \mathrm{~mm}$ wide and the inflorescence is simple or compound. A prolific nature (5000 seeds from a single plant) and a very short life cycle of C. iria help it to establish a second generation in the same growing season $[3,4]$. It is estimated that approximately $64 \%$ of rice yield reduction occurs due to this weed [5].

Weed management in the crop field is a challenging task in agriculture. Chemical herbicides are mainly preferred by the farmers to control weeds due to their higher efficacy, affordable cost and more rapid out return. The migration of labor away from agriculture to industries or other countries for employment is also a major concern for dependence in some countries [6]. However, the excessive use of synthetic herbicides can lead to an 
increase in the number of herbicide-resistant biotypes [7], low agricultural production, environmental pollution and health hazards $[8,9]$. On the other hand, the introduction of allelopathic plants or bio-herbicide develop from allelochemicals can play an important role as a substitute for the chemical dependence on synthetic chemical herbicides to control weeds in sustainable agriculture [10].

Invasive weed species have the potential to release allelopathic substances to the surrounding environments to suppress their neighboring competing plants [11-15]. Parthenium hysterophorus $\mathrm{L}$. has taken the shape of a noxious weed and is becoming a threat to crop production, animal husbandry and human health due to its strong allelopathic effects [16-19]. The isolation and identification of the allelopathic substances from P. hysterophorus could be used as a tool for the development of a natural-product-based herbicide for weed control.

Bioassays are generally designed to test the allelopathic properties of a plant species. However, a plant that shows strong phytotoxicity on the target plant species in laboratory conditions might not be so strong in the field condition due to the influence of several environmental factors $[20,21]$. In this context, two experiments were conducted in both laboratory and glasshouse conditions to evaluate the allelopathic properties of P. hysterophorus with a view to developing natural-product-based bioherbicides. The identification of its phytochemical constituents was analyzed by using LC-ESI-QTOF-MS=MS.

\section{Results}

\subsection{Laboratory Experiment}

Effect of Methanol Extracts on Germination and Initial Growth of $C$. iria

The results showed that $P$. hysterophorus extracts significantly $(p \leq 0.05)$ reduced the germination percentage as well as coleoptile and radicle length of $C$. iria (Table 1). The inhibitory activity was concentration-dependent. By the application of methanol extracts, the seed germination was significantly $(p \leq 0.05)$ reduced. No seed germination was recorded when $P$. hysterophorus extract was applied at $50 \mathrm{~g} \mathrm{~L}^{-1}$.

Parthenium hysterophorus extract decreased the coleoptile and radicle elongation of $C$. iria. The magnitude of inhibition increased with an increase in extract concentration. At a concentration of $50 \mathrm{~g} \mathrm{~L}^{-1}$ or above, P. hysterophorus extract reduced the coleoptile and radicle length of C. iria by $100 \%$.

Table 1. Effects of P. hysterophorus on germination, coleoptile and radicle length of C. iria.

\begin{tabular}{cccc}
\hline Dose $\left(\mathrm{g} \mathrm{L}^{-\mathbf{1}}\right)$ & Germination (\%) & Coleoptile Length (cm) & Radicle Length (cm) \\
\hline 0.00 & $100.00 \mathrm{a}(0)$ & $1.51 \mathrm{a}(0)$ & $1.66 \mathrm{a}(0)$ \\
6.25 & $80.00 \mathrm{~b}(20)$ & $1.20 \mathrm{~b}(20.72)$ & $1.10 \mathrm{~b}(33.68)$ \\
12.5 & $47.00 \mathrm{c}(53)$ & $0.86 \mathrm{c}(43.14)$ & $0.60 \mathrm{c}(64.02)$ \\
25 & $19.00 \mathrm{~d}(81)$ & $0.36 \mathrm{~d}(76.24)$ & $0.24 \mathrm{~d}(85.65)$ \\
50 & $0.00 \mathrm{e}(100)$ & $0.00 \mathrm{e}(100)$ & $0.00 \mathrm{e}(100)$ \\
100 & $0.00 \mathrm{e}(100)$ & $0.00 \mathrm{e}(100)$ & $0.00 \mathrm{e}(100)$ \\
\hline
\end{tabular}

Data are expressed as means. Means with same letters in the column for concentrations are not significantly different at $p>0.05$. Values inside the parenthesis are inhibition percentages relative to the control.

\subsection{Glasshouse Experiment}

\subsubsection{Effect of Methanol Extract on Plant Height, Leaf Area and Dry Weight of C. iria}

Table 2 showed the effect of P. hysterophorus methanol extract on the plant height, leaf area and dry weight of tested weeds. Dose-dependent inhibitory activity was also observed here. Parthenium hysterophorus showed significant inhibition on plant height at the highest concentration $\left(100 \mathrm{~g} \mathrm{~L}^{-1}\right)$. At the concentration of $100 \mathrm{~g} \mathrm{~L}^{-1}$, P. hysterophorus extract $44.40 \%$ inhibition was observed on the plant height of $C$. iria. A decline in leaf area of the tested weed was also observed with an increase in P. hysterophorus methanol extract concentration. The leaf area inhibition of $C$. iria ranged from 7.63 to $52.03 \%$ from $6.25 \mathrm{~g} \mathrm{~L}^{-1}$ to $100 \mathrm{~g} \mathrm{~L}^{-1}$ 
concentrations of $P$. hysterophorus extract. The control obtained the highest dry weight. The extract reduced $60.81 \%$ of the dry weight of $C$. iria at $100 \mathrm{~g} \mathrm{~L}^{-1}$ compared to the control.

Table 2. Effect of P. hysterophorus methanol extracts on the plant height $(\mathrm{cm})$, leaf area $\left(\mathrm{cm}^{2}\right)$ and $\operatorname{dry}$ weight $\left(\mathrm{g}\right.$ pot $\left.{ }^{-1}\right)$ of $C$. iria.

\begin{tabular}{cccc}
\hline Dose $\left(\mathbf{g ~ L}^{-\mathbf{1}}\right)$ & Plant Height & Leaf Area & Dry Weight \\
\hline 0 & $64.75 \mathrm{a}(0)$ & $151.05 \mathrm{a}(0)$ & $5.12 \mathrm{a}(0)$ \\
6.25 & $63.37 \mathrm{ab}(2.13)$ & $139.52 \mathrm{~b}(7.63)$ & $4.89 \mathrm{ab}(4.46)$ \\
12.5 & $62.02 \mathrm{ab}(4.20)$ & $132.24 \mathrm{c}(12.44)$ & $4.53 \mathrm{~b}(11.44)$ \\
25 & $57.42 \mathrm{~b}(11.29)$ & $115.22 \mathrm{~d}(23.70)$ & $3.86 \mathrm{c}(24.55)$ \\
50 & $50.31 \mathrm{c}(22.31)$ & $91.15 \mathrm{e}(39.63)$ & $3.00 \mathrm{~d}(41.20)$ \\
100 & $36.00 \mathrm{~d}(44.40)$ & $72.45 \mathrm{f}(52.03)$ & $2.00 \mathrm{e}(60.81)$ \\
\hline
\end{tabular}

Data are expressed as means. Means with same letters in the column for each extract concentrations are not significantly different at $p>0.05$. Values inside the parenthesis are inhibition percentages relative to the control.

2.2.2. Effect of Methanol Extract on Fv/Fm, Photosynthesis Rate, Stomatal Conductance and Transpiration Rate of C. iria

No significant difference was observed when C. iria was treated with 6.25 and $12.5 \mathrm{~g} \mathrm{~L}^{-1}$ of $P$. hysterophorus extract (Table 3). The extract reduced the Fv/Fm value by $46.32 \%$ at $100 \mathrm{~g} \mathrm{~L}^{-1}$. The significant effect of extracts concentrations was observed on the photosynthesis, stomatal conductance and transpiration rate of $C$. iria. The photosynthesis rate of $C$. iria was inhibited by $44.41 \%$ when treated with the highest concentrations $\left(100 \mathrm{~g} \mathrm{~L}^{-1}\right)$ of $P$. hysterophorus extract. The lowest stomatal conductance $\left(0.25 \mathrm{~mol} \mathrm{~m}^{-2} \mathrm{~s}^{-1}\right)$ was recorded at $100 \mathrm{~g} \mathrm{~L}^{-1}$, and the inhibition value was $39.63 \%$ (Table 4$)$. The lowest transpiration rate was observed at the highest concentration $\left(100 \mathrm{~g} \mathrm{~L}^{-1}\right)$, and the inhibition value was $40.98 \%$.

Table 3. Effects of $P$. hysterophorus methanol extract on $\mathrm{Fv} / \mathrm{Fm}$, photosynthesis rate $\left(\mu \mathrm{mol} \mathrm{m}{ }^{-2} \mathrm{~s}^{-1}\right)$, stomatal conductance $\left(\mathrm{mol} \mathrm{m} \mathrm{m}^{-2} \mathrm{~s}^{-1}\right)$ and transpiration rate $\left(\mathrm{mmol} \mathrm{m}^{-2} \mathrm{~s}^{-1}\right)$ of C. iria.

\begin{tabular}{ccccc}
\hline $\begin{array}{c}\text { Dose } \\
\left(\mathbf{g ~ L}^{-\mathbf{1}}\right)\end{array}$ & Fv/Fm & Photosynthesis Rate & Stomatal Conductance & Transpiration Rate \\
\hline 0 & $1.47 \mathrm{a}(0)$ & $45.14 \mathrm{a}(0)$ & $0.42 \mathrm{a}(0)$ & $11.50 \mathrm{a}(0)$ \\
6.25 & $1.41 \mathrm{a}(3.90)$ & $43.50 \mathrm{ab}(3.64)$ & $0.41 \mathrm{ab}(3.43)$ & $10.83 \mathrm{~b}(5.82)$ \\
12.5 & $1.34 \mathrm{a}(8.56)$ & $42.50 \mathrm{ab}(5.86)$ & $0.40 \mathrm{ab}(6.04)$ & $10.41 \mathrm{c}(9.52)$ \\
25 & $1.20 \mathrm{ab}(17.84)$ & $40.00 \mathrm{~b}(11.37)$ & $0.38 \mathrm{~b}(10.07)$ & $9.35 \mathrm{~d}(18.69)$ \\
50 & $1.08 \mathrm{ab}(26.19)$ & $35.29 \mathrm{c}(21.86)$ & $0.34 \mathrm{c}(20.31)$ & $8.20 \mathrm{e}(28.67)$ \\
100 & $0.79 \mathrm{~b}(46.32)$ & $25.13 \mathrm{~d}(44.41)$ & $0.25 \mathrm{~d}(39.63)$ & $6.79 \mathrm{f}(40.98)$ \\
\hline
\end{tabular}

Data are expressed as means. Means with same letters in the column for each extract concentrations are not significantly different at $p>0.05$. Values inside the parenthesis are inhibition percentages relative to the control.

Table 4. LC-MS profile of methanol extract of P. hysterophorus.

\begin{tabular}{|c|c|c|c|c|c|}
\hline $\begin{array}{l}\text { Sl. } \\
\text { No }\end{array}$ & RT (min) & Proposed Compound & $\begin{array}{l}\text { Molecular } \\
\text { Formula }\end{array}$ & $\begin{array}{l}\text { Mass Fragment } \\
(\mathrm{m} / \mathrm{z})\end{array}$ & Polarity \\
\hline 1 & 1.436 & Valine & $\mathrm{C}_{5} \mathrm{H}_{11} \mathrm{NO}_{2}$ & 117.0802 & Positive \\
\hline 2 & 1.418 & Glyceryl sulfoquinovoside & $\mathrm{C}_{9} \mathrm{H}_{18} \mathrm{O}_{10} \mathrm{~S}$ & 318.063 & Negative \\
\hline 3 & 1.575 & Lotaustralin & $\mathrm{C}_{11} \mathrm{H}_{19} \mathrm{NO}_{6}$ & 261.1215 & Positive \\
\hline 4 & 3.162 & Trazolopride & $\mathrm{C}_{20} \mathrm{H}_{23} \mathrm{~N}_{5} \mathrm{O}_{2}$ & 365.1851 & Positive \\
\hline 5 & 3.571 & Pirenzepine & $\mathrm{C}_{19} \mathrm{H}_{21} \mathrm{~N}_{5} \mathrm{O}_{2}$ & 351.1694 & Positive \\
\hline 6 & 3.92 & $\begin{array}{l}\text { 1-Cyclopropyl-3-[[1-(4-hydroxybutyl)benzimidazol-2- } \\
\text { yl]methyl]imidazo [4,5-c]pyridin-2-one }\end{array}$ & $\mathrm{C}_{21} \mathrm{H}_{23} \mathrm{~N}_{5} \mathrm{O}_{2}$ & 377.1848 & Positive \\
\hline 7 & 4.239 & Umbelliferone & $\mathrm{C}_{9} \mathrm{H}_{6} \mathrm{O}_{3}$ & 162.0317 & Positive \\
\hline 8 & 4.244 & Quinic Acid & $\mathrm{C}_{7} \mathrm{H}_{12} \mathrm{O}_{6}$ & 192.0638 & Negative \\
\hline 9 & 4.941 & Atevirdine & $\mathrm{C}_{21} \mathrm{H}_{25} \mathrm{~N}_{5} \mathrm{O}_{2}$ & 379.2002 & Positive \\
\hline
\end{tabular}


Table 4. Cont

\begin{tabular}{|c|c|c|c|c|c|}
\hline $\begin{array}{l}\text { Sl. } \\
\text { No }\end{array}$ & RT (min) & Proposed Compound & $\begin{array}{l}\text { Molecular } \\
\text { Formula }\end{array}$ & $\begin{array}{l}\text { Mass Fragment } \\
\qquad(\mathrm{m} / \mathrm{z})\end{array}$ & Polarity \\
\hline 10 & 5.253 & Dihydrophaseic acid 4-O-beta-D-glucoside & $\mathrm{C}_{21} \mathrm{H}_{32} \mathrm{O}_{10}$ & 444.1998 & Negative \\
\hline 11 & 5.536 & 2-(2-Ethoxyethoxy)ethanol;4-methylbenzenesulfonic acid & $\mathrm{C}_{13} \mathrm{H}_{22} \mathrm{O}_{6} \mathrm{~S}$ & 306.1136 & Negative \\
\hline 12 & 5.475 & $\begin{array}{c}\text { 4-Azidobenzyl benzyl 1,4-butanediylbiscarbamate } \\
\text { 4-(N-hydroxyamino)-2r-isobutyl-2s- }(2-\end{array}$ & $\mathrm{C}_{20} \mathrm{H}_{23} \mathrm{~N}_{5} \mathrm{O}_{4}$ & 397.175 & Positive \\
\hline 13 & 5.823 & $\begin{array}{l}\text { Thienylthiomethyl)succinyl-L-Phenylalanine-N- } \\
\text { Methylamide }\end{array}$ & $\mathrm{C}_{20} \mathrm{H}_{31} \mathrm{NO}_{3} \mathrm{~S}_{2}$ & 397.176 & Positive \\
\hline 14 & 6.08 & Branaplam & $\mathrm{C}_{22} \mathrm{H}_{27} \mathrm{~N}_{5} \mathrm{O}_{2}$ & 393.2162 & Positive \\
\hline 15 & 6.257 & Pulchellamine G & $\mathrm{C}_{21} \mathrm{H}_{31} \mathrm{NO}_{6}$ & 393.2151 & Positive \\
\hline 16 & 6.503 & Hymenoxynin & $\mathrm{C}_{21} \mathrm{H}_{34} \mathrm{O}_{9}$ & 430.2208 & Negative \\
\hline 17 & 6.939 & Chlorogenic acid & $\mathrm{C}_{16} \mathrm{H}_{18} \mathrm{O}_{9}$ & 354.0957 & Negative \\
\hline 18 & 7.006 & Parthenin & $\mathrm{C}_{15} \mathrm{H}_{18} \mathrm{O}_{4}$ & 262.1202 & Positive \\
\hline 19 & 7.006 & Gaillardilin & $\mathrm{C}_{17} \mathrm{H}_{22} \mathrm{O}_{6}$ & 322.1415 & Positive \\
\hline 20 & 7.006 & Dehydroleucodine & $\mathrm{C}_{15} \mathrm{H}_{16} \mathrm{O}_{3}$ & 244.1095 & Positive \\
\hline 21 & 7.264 & N-Propyl-3-(1,3-thiazol-2-yl)thian-3-amine & $\mathrm{C}_{11} \mathrm{H}_{18} \mathrm{~N}_{2} \mathrm{~S}_{2}$ & 242.0928 & Positive \\
\hline 22 & 7.266 & Oleacein & $\mathrm{C}_{17} \mathrm{H}_{20} \mathrm{O}_{6}$ & 320.1252 & Positive \\
\hline 23 & 7.49 & Bendazac lysine & $\mathrm{C}_{22} \mathrm{H}_{28} \mathrm{~N}_{4} \mathrm{O}_{5}$ & 428.2053 & Negative \\
\hline 24 & 7.641 & Lajollamide A & $\mathrm{C}_{30} \mathrm{H}_{55} \mathrm{~N}_{5} \mathrm{O}_{5}$ & 565.4206 & Positive \\
\hline 25 & 7.673 & Isochlorogenic acid $\mathrm{A}$ & $\mathrm{C}_{25} \mathrm{H}_{24} \mathrm{O}_{12}$ & 516.127 & Negative \\
\hline 26 & 7.673 & Chlorogenic acid & $\mathrm{C}_{16} \mathrm{H}_{18} \mathrm{O}_{9}$ & 354.0958 & Negative \\
\hline 27 & 7.897 & $\begin{array}{l}\text { 4-[(6-Chloro-2-naphthalenyl)sulfonyl]-1-[[1-(4-pyridinyl)- } \\
\text { 4- piperidinyl]methyl]-2 piperazinecarboxylic acid }\end{array}$ & $\mathrm{C}_{27} \mathrm{H}_{41} \mathrm{ClN}_{4} \mathrm{O}_{6}$ & 552.2699 & Positive \\
\hline 28 & 7.905 & $\begin{array}{l}\text { N-Chloro-9-(diaminomethylideneamino)-3- } \\
\text { hydroxynonanamide }\end{array}$ & $\mathrm{C}_{10} \mathrm{H}_{21} \mathrm{ClN}_{4} \mathrm{O}_{2}$ & 264.1358 & Positive \\
\hline 29 & 7.908 & 1-(N-6-Amino-n-hexyl)carbamoylimidazole & $\mathrm{C}_{10} \mathrm{H}_{19} \mathrm{ClN}_{4} \mathrm{O}$ & 246.1253 & Positive \\
\hline 30 & 8.042 & 2,4-Toluene Diisocyanate Dimer & $\mathrm{C}_{18} \mathrm{H}_{12} \mathrm{~N}_{4} \mathrm{O}_{4}$ & 348.0862 & Positive \\
\hline 31 & 8.044 & Alaptide & $\mathrm{C}_{9} \mathrm{H}_{14} \mathrm{~N}_{2} \mathrm{O}_{2}$ & 182.1063 & Positive \\
\hline 32 & 8.05 & Carbocyclic- $3^{\prime}$-amino-ara-adenosine & $\mathrm{C}_{11} \mathrm{H}_{16} \mathrm{~N}_{6} \mathrm{O}_{2}$ & 264.1339 & Positive \\
\hline 33 & 8.054 & Tris(pyrazolyl)ethane & $\mathrm{C}_{11} \mathrm{H}_{12} \mathrm{~N}_{6}$ & 228.1118 & Positive \\
\hline 34 & 8.055 & Descyclopropyl Abacavir & $\mathrm{C}_{11} \mathrm{H}_{14} \mathrm{~N}_{6} \mathrm{O}$ & 246.1225 & Positive \\
\hline 35 & 8.058 & 1-Boc-3-oxopiperazine & $\mathrm{C}_{9} \mathrm{H}_{16} \mathrm{~N}_{2} \mathrm{O}_{3}$ & 200.1162 & Positive \\
\hline 36 & 8.13 & Teroxalene hydrochloride & $\mathrm{C}_{28} \mathrm{H}_{42} \mathrm{Cl}_{2} \mathrm{~N}_{2} \mathrm{OS}$ & 524.2364 & Positive \\
\hline 37 & 8.132 & $\begin{array}{l}\text { Ethane;(3-oxo-6'-sulfanylcarbonyloxyspiro [2 -benzofuran- } \\
\text { 1,9'-xanthene]-3'-yl)oxymethanethioicS-acid;propane }\end{array}$ & $\mathrm{C}_{31} \mathrm{H}_{38} \mathrm{O}_{7} \mathrm{~S}_{2}$ & 586.206 & Positive \\
\hline 38 & 8.133 & (2-Aminoethylamino) 2,2-diaminooxyacetate & $\mathrm{C}_{4} \mathrm{H}_{12} \mathrm{~N}_{4} \mathrm{O}_{4}$ & 180.0845 & Positive \\
\hline 39 & 8.134 & $\begin{array}{l}\text { N-[(S)-2-Benzo[1,3]dioxol-5-yl-4-(4-phenyl-piperidin-1- } \\
\text { yl)-butyl]-N-methyl-benzenesulfonamide }\end{array}$ & $\mathrm{C}_{29} \mathrm{H}_{34} \mathrm{~N}_{2} \mathrm{O}_{4} \mathrm{~S}$ & 506.2237 & Positive \\
\hline 40 & 8.135 & 3-Diazo-1-hexylsulfanyl-1-methylurea & $\mathrm{C}_{8} \mathrm{H}_{16} \mathrm{~N}_{4} \mathrm{OS}$ & 216.1055 & Positive \\
\hline 41 & 8.135 & Ethylene oxide-b-maleic hydrazide & $\mathrm{C}_{6} \mathrm{H}_{12} \mathrm{~N}_{8} \mathrm{O}_{3}$ & 244.103 & Positive \\
\hline 42 & 8.136 & N-[3-(1H-Imidazol-4-yl)propyl]-N'-methylthiourea & $\mathrm{C}_{8} \mathrm{H}_{14} \mathrm{~N}_{4} \mathrm{~S}$ & 198.0952 & Positive \\
\hline 43 & 8.136 & 1-Methylpiperazine-1,4-Diium Bis & $\mathrm{C}_{5} \mathrm{H}_{14} \mathrm{~N}_{4} \mathrm{O}_{6}$ & 226.0914 & Positive \\
\hline 44 & 8.136 & 3-(2-Methylpropylthio)-1H-1,2,4-triazol-5-amine & $\mathrm{C}_{6} \mathrm{H}_{12} \mathrm{~N}_{4} \mathrm{~S}$ & 172.0801 & Positive \\
\hline 45 & 8.136 & Benzylamidinoisothiourea & $\mathrm{C}_{9} \mathrm{H}_{12} \mathrm{~N}_{4} \mathrm{~S}$ & 208.0792 & Positive \\
\hline 46 & 8.136 & 1-Amino-3-(propylamino)thiourea & $\mathrm{C}_{4} \mathrm{H}_{12} \mathrm{~N}_{4} \mathrm{~S}$ & 148.0798 & Positive \\
\hline 47 & 8.136 & 9-hydroxyellipticine & $\mathrm{C}_{17} \mathrm{H}_{14} \mathrm{~N}_{2} \mathrm{O}$ & 262.1122 & Positive \\
\hline 48 & 8.136 & 4-Phenylamino-3-quinolinecarbonitrile deriv. 28 & $\mathrm{C}_{27} \mathrm{H}_{30} \mathrm{Cl}_{2} \mathrm{~N}_{4} \mathrm{O}_{4}$ & 544.16 & Positive \\
\hline 49 & 8.136 & 1-(3-ethyl-1,2,4-thiadiazol-5-yl)azetidin-3-amine & $\mathrm{C}_{7} \mathrm{H}_{12} \mathrm{~N}_{4} \mathrm{~S}$ & 184.0793 & Positive \\
\hline 50 & 8.413 & $\begin{array}{l}\text { 1,8,15,22,29,36-Hexaazacyclodotetracontane- } \\
\text { 2,7,16,21,30,35-hexone }\end{array}$ & $\mathrm{C}_{36} \mathrm{H}_{66} \mathrm{~N}_{6} \mathrm{O}_{6}$ & 678.504 & Positive \\
\hline 51 & 8.415 & 2,4,6-tris(3-methylbutoxy)-1,3,5-triazine & $\mathrm{C}_{18} \mathrm{H}_{33} \mathrm{~N}_{3} \mathrm{O}_{3}$ & 339.2522 & Positive \\
\hline 52 & 8.435 & Arginyl-tyrosyl-aspartic acid & $\mathrm{C}_{19} \mathrm{H}_{28} \mathrm{~N}_{6} \mathrm{O}_{7}$ & 452.2022 & Positive \\
\hline 53 & 8.636 & 8-(2,4,6-Trimethoxyphenyl)-9H-purine-2,6-diamine & $\mathrm{C}_{14} \mathrm{H}_{16} \mathrm{~N}_{6} \mathrm{O}_{3}$ & 316.1282 & Positive \\
\hline 54 & 8.818 & Dimethyl 2-(heptane-1-sulfonyl)butanedioate & $\mathrm{C}_{13} \mathrm{H}_{24} \mathrm{O}_{6} \mathrm{~S}$ & 308.1298 & Negative \\
\hline 55 & 8.721 & AC-Ala-gln-ala-pna & $\mathrm{C}_{19} \mathrm{H}_{26} \mathrm{~N}_{6} \mathrm{O}_{7}$ & 450.1864 & Positive \\
\hline 56 & 9.065 & Laciniatin & $\mathrm{C}_{17} \mathrm{H}_{14} \mathrm{O}_{8}$ & 346.0693 & Positive \\
\hline 57 & 9.067 & 2-[(3,5-Dinitrobenzoyl)amino]benzoic acid & $\mathrm{C}_{14} \mathrm{H}_{9} \mathrm{~N}_{3} \mathrm{O}_{7}$ & 331.0461 & Negative \\
\hline
\end{tabular}


Table 4. Cont.

\begin{tabular}{|c|c|c|c|c|c|}
\hline $\begin{array}{l}\text { Sl. } \\
\text { No }\end{array}$ & RT (min) & Proposed Compound & $\begin{array}{l}\text { Molecular } \\
\text { Formula }\end{array}$ & $\begin{array}{l}\text { Mass Fragment } \\
(\mathrm{m} / \mathrm{z})\end{array}$ & Polarity \\
\hline 58 & 9.243 & $\begin{array}{l}\text { 3-Ethyl-1-propyl-8-(1H-pyrazol-4-yl)-1H-purine- } \\
2,6(3 \mathrm{H}, 7 \mathrm{H}) \text {-dione }\end{array}$ & $\mathrm{C}_{13} \mathrm{H}_{16} \mathrm{~N}_{6} \mathrm{O}_{2}$ & 288.134 & Positive \\
\hline 59 & 11.645 & Apnea & $\mathrm{C}_{18} \mathrm{H}_{22} \mathrm{~N}_{6} \mathrm{O}_{4}$ & 386.1696 & Positive \\
\hline 60 & 11.844 & Thyroliberin N-ethylamide & $\mathrm{C}_{18} \mathrm{H}_{26} \mathrm{~N}_{6} \mathrm{O}_{4}$ & 390.2011 & Positive \\
\hline 61 & 11.996 & Hexadecasphinganine & $\mathrm{C}_{16} \mathrm{H}_{35} \mathrm{NO}_{2}$ & 273.2672 & Positive \\
\hline 62 & 12.034 & Phytosphingosine & $\mathrm{C}_{18} \mathrm{H}_{39} \mathrm{NO}_{3}$ & 317.2935 & Positive \\
\hline 63 & 12.176 & Dihydroxyethyllauramine oxide & $\mathrm{C}_{16} \mathrm{H}_{35} \mathrm{NO}_{3}$ & 289.262 & Positive \\
\hline 64 & 12.193 & Lauramine oxide & $\mathrm{C}_{14} \mathrm{H}_{31} \mathrm{NO}$ & 229.2405 & Positive \\
\hline 65 & 12.308 & Rishitin & $\mathrm{C}_{14} \mathrm{H}_{22} \mathrm{O}_{2}$ & 222.161 & Negative \\
\hline 66 & 12.316 & Dioctylnitrosamine & $\mathrm{C}_{16} \mathrm{H}_{34} \mathrm{~N}_{2} \mathrm{O}$ & 270.2673 & Positive \\
\hline 67 & 12.343 & Dodecylacrylamide & $\mathrm{C}_{15} \mathrm{H}_{29} \mathrm{NO}$ & 239.2251 & Positive \\
\hline 68 & 12.349 & Tetrabutylurea & $\mathrm{C}_{17} \mathrm{H}_{36} \mathrm{~N}_{2} \mathrm{O}$ & 284.2832 & Positive \\
\hline 69 & 12.703 & Aminopregnane & $\mathrm{C}_{21} \mathrm{H}_{37} \mathrm{~N}$ & 303.2934 & Positive \\
\hline 70 & 12.778 & Tridecylglycerol & $\mathrm{C}_{16} \mathrm{H}_{34} \mathrm{O}_{3}$ & 274.2512 & Positive \\
\hline 71 & 13.164 & 2,3,3-Tris(1,2-diaminoethyl)-2-ethylhexanoic acid & $\mathrm{C}_{14} \mathrm{H}_{34} \mathrm{~N}_{6} \mathrm{O}_{2}$ & 318.2769 & Positive \\
\hline 72 & 13.633 & 4-dodecylbenzenesulfonic acid & $\mathrm{C}_{18} \mathrm{H}_{30} \mathrm{O}_{3} \mathrm{~S}$ & 326.1916 & Negative \\
\hline 73 & 14.691 & Angoletin & $\mathrm{C}_{18} \mathrm{H}_{20} \mathrm{O}_{4}$ & 300.1357 & Positive \\
\hline 74 & 14.694 & Phthalic anhydride & $\mathrm{C}_{8} \mathrm{H}_{4} \mathrm{O}_{3}$ & 148.069 & Positive \\
\hline 75 & 15.406 & Eicosasphinganine & $\mathrm{C}_{20} \mathrm{H}_{43} \mathrm{NO}_{2}$ & 329.3298 & Positive \\
\hline 76 & 16.483 & Lauryl sulfate & $\mathrm{C}_{12} \mathrm{H}_{26} \mathrm{O}_{4} \mathrm{~S}$ & 266.1551 & Negative \\
\hline 77 & 16.957 & Dodecandial-disemicarbazon & $\mathrm{C}_{14} \mathrm{H}_{28} \mathrm{~N}_{6} \mathrm{O}_{2}$ & 312.2282 & Positive \\
\hline 78 & 18.267 & Benzenesulfonic acid, tridecyl- & $\mathrm{C}_{19} \mathrm{H}_{32} \mathrm{O}_{3} \mathrm{~S}$ & 340.2072 & Negative \\
\hline 79 & 19.135 & 3-[5-(3-Dimethylamino-1,2,4-thiadiazol)-yl] quinuclidine & $\mathrm{C}_{11} \mathrm{H}_{18} \mathrm{~N}_{4} \mathrm{~S}$ & 238.125 & Positive \\
\hline 80 & 19.496 & Benzenesulfonic acid, undecyl- & $\mathrm{C}_{17} \mathrm{H}_{28} \mathrm{O}_{3} \mathrm{~S}$ & 312.176 & Negative \\
\hline 81 & 19.918 & N,N-bis(2-hydroxyethyl)stearylamine & $\mathrm{C}_{22} \mathrm{H}_{47} \mathrm{NO}_{2}$ & 357.3609 & Positive \\
\hline 82 & 20.245 & Benzoyl benzenecarboperoxoate;dodecane-1-thiol;toluene & $\mathrm{C}_{33} \mathrm{H}_{44} \mathrm{O}_{4} \mathrm{~S}$ & 536.2965 & Positive \\
\hline
\end{tabular}

\subsection{Identification of Phytotoxic Components from Methanol Extract of P. hysterophorus}

LC-MS analyses of $P$. hysterophorus methanol extract revealed the presence of 82 known compounds that appeared between 1 and 20 mins. The list of proposed compounds with their retention times, molecular formula, polarity and mass fragment $(\mathrm{m} / \mathrm{z})$ is shown in Table 4 . For most of the constituents, $[\mathrm{M}-\mathrm{H}]^{+}$and $[\mathrm{M}-\mathrm{H}]^{-}$ions were observed. The total ion current chromatography in positive and negative ESI mode is shown in Figures 1 and 2. Eight amino acids (Valine, Lajollamide A, Alaptide, Arginyl-tyrosylaspartic acid, Thyroliberin N-ethylamide, Hexadecasphinganine, Phytosphingosine and Eicosasphinganine) were identified, which usually provides $[\mathrm{M}-\mathrm{H}]^{+}$ions as the best peak positive ESI mode. The amino acids were identified at 1.436, 7.641, 8.004, 8.435, 11.844, $11.996,12.034,15.406 \mathrm{~min}$, with 117.0802, 565.4206, 182.1063, 452.2022, 390.2011, 273.2672, $317.2935,329.3298 \mathrm{~m} / \mathrm{z}$, respectively, in the positive ionization mode. A total of seven phenolic compounds (Umbelliferone, Quinic Acid, Chlorogenic acid, Oleacein, Isochlorogenic acid A, Laciniatinand Phthalic anhydride) and three terpenoids (Parthenin, Dehydroleucodine and Rishitin) were also identified. Among the phenolic compounds, chlorogenic acid $\left(\mathrm{C}_{16} \mathrm{H}_{18} \mathrm{O}_{9}\right)$ was detected with its $[\mathrm{M}-\mathrm{H}]^{-}$ion at $6.939 \mathrm{~min}$ with $354.0957 \mathrm{~m} / \mathrm{z}$. In positive ionization mode, parthenin $\left(\mathrm{C}_{15} \mathrm{H}_{18} \mathrm{O}_{4}\right)$ was detected at 7.006 min with 262.1202 $\mathrm{m} / \mathrm{z}$. A fragment ion at $262.1122 \mathrm{~m} / \mathrm{z}$ was displayed for 9-hydroxyellipticine (alkaloid) in positive ionization mode at $8.136 \mathrm{~min}$. A number of other organic compounds were also detected in P. hysterophorus (Table 4). Descyclopropyl Abacavir $\left(\mathrm{C}_{11} \mathrm{H}_{14} \mathrm{~N}_{6} \mathrm{O}\right)$ is a carbohydrate and was detected from the extract at $8.055 \mathrm{~min} 246.1225 \mathrm{~m} / \mathrm{z}$. At $229.24 \mathrm{~m} / \mathrm{z}$, Lauramine oxide $\left(\mathrm{C}_{14} \mathrm{H}_{31} \mathrm{NO}\right)$ was identified as a detergent at $12.193 \mathrm{~min}$. Glycolipid (Glyceryl sulfoquinovoside, $\mathrm{C}_{9} \mathrm{H}_{18} \mathrm{O}_{10} \mathrm{~S}$ ) and glycoside (Dihydrophaseic acid 4-O-beta-Dglucoside, $\mathrm{C}_{21} \mathrm{H}_{32} \mathrm{O}_{10}$ ) were identified at 1.418 and $5.253 \mathrm{~min}$ with 318.063 and 444.1998 $\mathrm{m} / \mathrm{z}$, respectively in the negative ionization mode. One ketone (Angoletin, $\mathrm{C}_{18} \mathrm{H}_{20} \mathrm{O}_{4}$ ) was also identified in the positive ionization mode at 14.691 with $300.1357 \mathrm{~m} / \mathrm{z}$. Two 
sulfonic acids, namely, 4-dodecylbenzenesulfonic acid $\left(\mathrm{C}_{18} \mathrm{H}_{30} \mathrm{O}_{3} \mathrm{~S}\right)$ and Benzenesulfonic acid, tridecyl- $\left(\mathrm{C}_{19} \mathrm{H}_{32} \mathrm{O}_{3} \mathrm{~S}\right)$ at 13.633 and $18.267 \mathrm{~min}$ with 326.1916 and $312.2282 \mathrm{~m} / \mathrm{z}$ in negative and positive ionization modes, respectively.

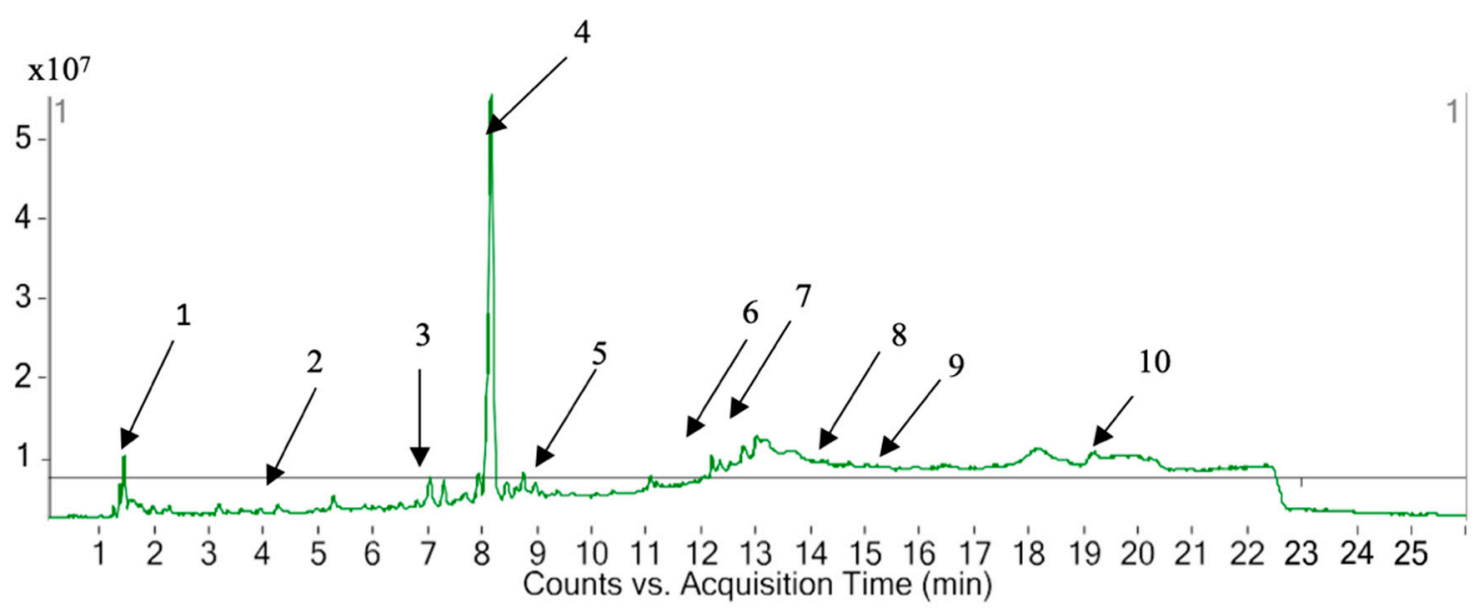

Figure 1. LC-MS chromatograms chemical compounds of P. hysterophorus in the positive ion mode (1. Valine, 2. umbelliferone, 3. parthenin, 4. 9-hydroxyellipticine, 5. laciniatin, 6. phytosphingosine, 7. tridecylglycerol, 8. phthalic anhydride, 9. eicosasphinganine, 10. N,N-bis (2-hydroxyethyl) stearylamine).

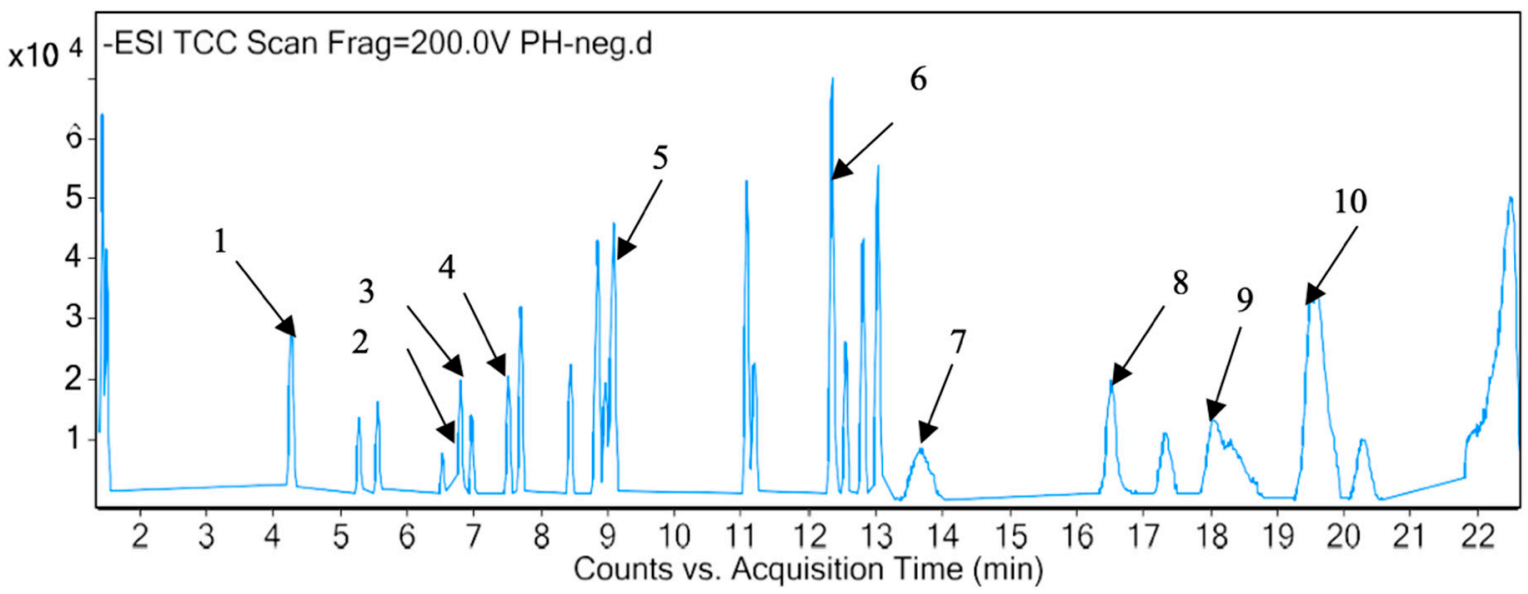

Figure 2. LC-MS chromatograms chemical compounds of P. hysterophorus in the negative ion mode (1. Quinic acid, 2. hymonoxynin, 3. chlorogenic acid, 4. isochlorogenic acid, 5. laciniatin, 6. Rishitin, 7. 4-dodecylbenzenesulfonic acid, 8. lauryl sulfate, 9. tridecyl-benzenesulfonic acid, 10. 4-undecyl benzene sulfonic acid).

\subsection{Efficacy of P. hysterophorus Extract in Comparison with Commercial Herbicides}

All treatments had significant effects $(p \leq 0.05)$ on plant height and fresh and dry weight (Table 5). The phytotoxicity effects of $P$. hysterophorus and synthetic herbicide on A. conyzoides, $C$. iria and $O$. sativa were evaluated based on visual observation at 21 days after spray (Table 5). The visual injury of $A$. conyzoides was higher compared to $C$. iria and $O$. sativa at the applied concentrations of $P$. hysterophorus methanol extract. At the highest concentration $\left(80 \mathrm{~g} \mathrm{~L}^{-1}\right), A$. conyzoides, $C$. iria and $O$. sativa were injured severely with an injury rating scale of 9.00, 5.25 and 4.50, respectively. Cyperus iria and O. sativa were alive and showed either green foliage or minor chlorosis or minor leaf curling at the lowest concentration $\left(20 \mathrm{~g} \mathrm{~L}^{-1}\right)$. All tested weeds died after treated with synthetic herbicide (glyphosate and glufosinate ammonium). However, only A. conyzoides died when P. hysterophorus was sprayed at $80 \mathrm{~g} \mathrm{~L}^{-1}$ (Figures 3 and 4). 
Table 5. Effect of P. hysterophorus on the visual injury, plant height, fresh weight and dry weight of $A$. conyzoides, C. iria and O. sativa.

\begin{tabular}{|c|c|c|c|c|c|c|c|}
\hline & \multirow[t]{2}{*}{ Tested Weeds } & \multicolumn{4}{|c|}{ P. hysterophorus } & \multicolumn{2}{|c|}{ Synthetic Herbicides } \\
\hline & & $0 \mathrm{~g} \mathrm{~L}^{-1}$ & $20 \mathrm{~g} \mathrm{~L}^{-1}$ & $40 \mathrm{~g} \mathrm{~L}^{-1}$ & $80 \mathrm{~g} \mathrm{~L}^{-1}$ & Glyphosate & Glufosinate-Ammonium \\
\hline \multirow{3}{*}{ Visual injury (Scale) } & A. conyzoides & $1.00 \mathrm{~d}$ & $2.75 c$ & $5.50 \mathrm{~b}$ & $9.00 \mathrm{a}$ & $9.00 \mathrm{a}$ & $9.00 \mathrm{a}$ \\
\hline & C. iria & $1.00 \mathrm{e}$ & $2.50 \mathrm{~d}$ & $4.00 \mathrm{c}$ & $5.25 \mathrm{~b}$ & $9.00 \mathrm{a}$ & $9.00 \mathrm{a}$ \\
\hline & O. sativa & $1.00 \mathrm{e}$ & $2.25 \mathrm{~d}$ & $3.00 \mathrm{c}$ & $4.50 \mathrm{~b}$ & $9.00 \mathrm{a}$ & $9.00 \mathrm{a}$ \\
\hline \multirow{6}{*}{ Plant height (cm) } & & $32.00 \mathrm{a}$ & $24.62 b$ & $14.62 \mathrm{c}$ & $0.00 \mathrm{~d}$ & $0.00 \mathrm{~d}$ & $0.00 \mathrm{~d}$ \\
\hline & A. conyzoides & $(0)$ & $(23.02)$ & $(54.32)$ & $(100)$ & $(100)$ & $(100)$ \\
\hline & & $64.75 a$ & $55.75 b$ & $44.25 c$ & $37.00 \mathrm{~d}$ & $0.00 \mathrm{e}$ & $0.00 \mathrm{e}$ \\
\hline & C. $ı$ ria & $(0)$ & $(13.58)$ & $(37.71)$ & $(42.97)$ & $(100)$ & $(100)$ \\
\hline & & $67.00 \mathrm{a}$ & $58.50 \mathrm{~b}$ & $49.50 c$ & $39.53 d$ & $0.00 \mathrm{e}$ & $0.00 \mathrm{e}$ \\
\hline & O. sativa & $(0)$ & $(12.68)$ & $(26.08)$ & $(41.02)$ & $(100)$ & $(100)$ \\
\hline \multirow{6}{*}{ Fresh weight $\left(\mathrm{g} \mathrm{pot}^{-1}\right)$} & & $26.45 a$ & $18.34 b$ & $3.14 \mathrm{c}$ & $0.45 \mathrm{~d}$ & $0.22 \mathrm{~d}$ & $0.27 \mathrm{~d}$ \\
\hline & A. conyzordes & $(0)$ & $(30.66)$ & $(88.10)$ & (98.28) & (99.17) & $(98.96)$ \\
\hline & C ixig & $25.95 a$ & $20.21 b$ & $15.70 \mathrm{c}$ & $12.80 \mathrm{~d}$ & $0.30 \mathrm{e}$ & $0.50 \mathrm{e}$ \\
\hline & C. 2 ria & $(0)$ & $(22.10)$ & $(39.45)$ & $(50.60)$ & $(98.86)$ & $(98.08)$ \\
\hline & O satizia & $12.70 \mathrm{a}$ & $8.89 b$ & $6.99 c$ & $5.44 \mathrm{~d}$ & $0.14 \mathrm{e}$ & $0.19 \mathrm{e}$ \\
\hline & U. sativa & $(0)$ & $(29.97)$ & $(44.93)$ & $(57.13)$ & $(98.92)$ & $(98.48)$ \\
\hline \multirow{6}{*}{ Dry weight $\left(\mathrm{g} \mathrm{pot}^{-1}\right)$} & A. conyzoides & $5.13 a$ & $3.04 \mathrm{~b}$ & $0.50 \mathrm{c}$ & $0.07 \mathrm{c}$ & $0.03 c$ & $0.05 c$ \\
\hline & A. conyzoudes & $(0)$ & $(40.78)$ & (90.36) & (98.63) & (99.42) & (99.08) \\
\hline & & $6.29 a$ & $4.95 b$ & $3.98 \mathrm{c}$ & $2.28 \mathrm{~d}$ & $0.06 \mathrm{e}$ & $0.10 \mathrm{e}$ \\
\hline & C. $\operatorname{ria}$ & $(0)$ & (21.12) & (36.53) & $(63.80)$ & (98.97) & $(98.43)$ \\
\hline & $\Omega$ satiza & $3.36 \mathrm{a}$ & $2.25 b$ & $1.75 b c$ & $1.24 \mathrm{c}$ & $0.03 \mathrm{~d}$ & $0.04 \mathrm{~d}$ \\
\hline & O. sattva & $(0)$ & (32.27) & (47.49) & $(62.76)$ & (99.05) & (98.77) \\
\hline
\end{tabular}

Data are expressed as means. Means with same letters in the row are not significantly different at $p<0.05$. Values inside the parenthesis are inhibition percentages relative to the control.

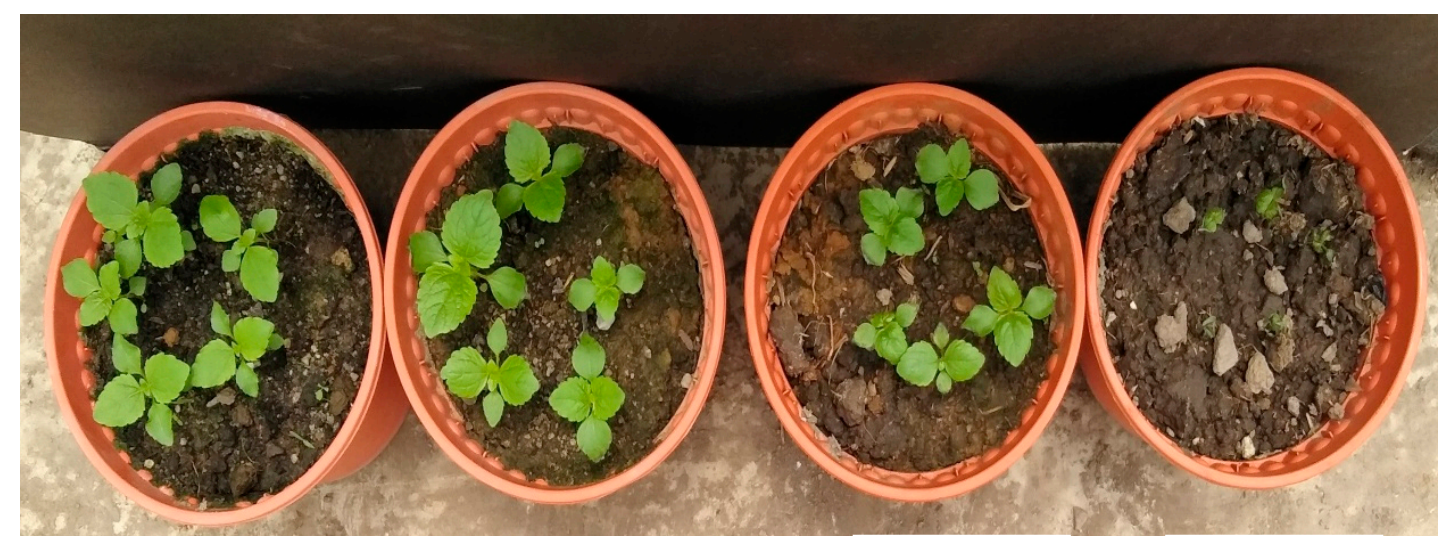
Control
(Untreated)
$20 \mathrm{~g} \mathrm{~L}^{-1}$
$40 \mathrm{~g} \mathrm{~L}^{-1}$
$80 \mathrm{~g} \mathrm{~L}^{-1}$

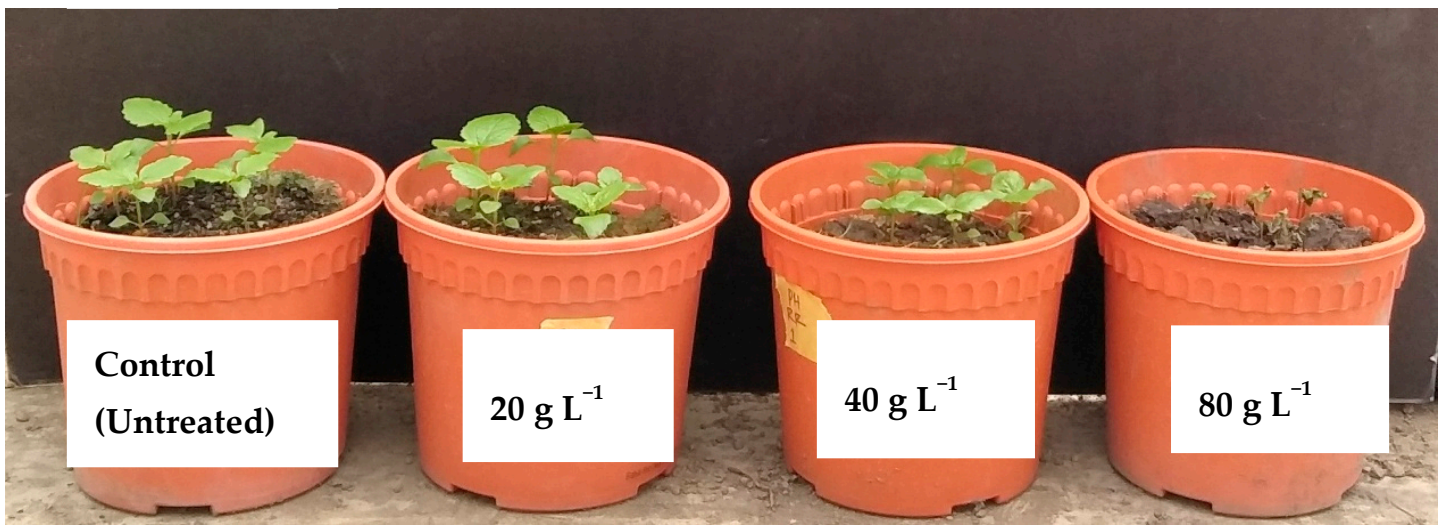

Figure 3. Effect of $P$. hysterophorus extract on A. conyzoides at $24 \mathrm{~h}$ after spray. 


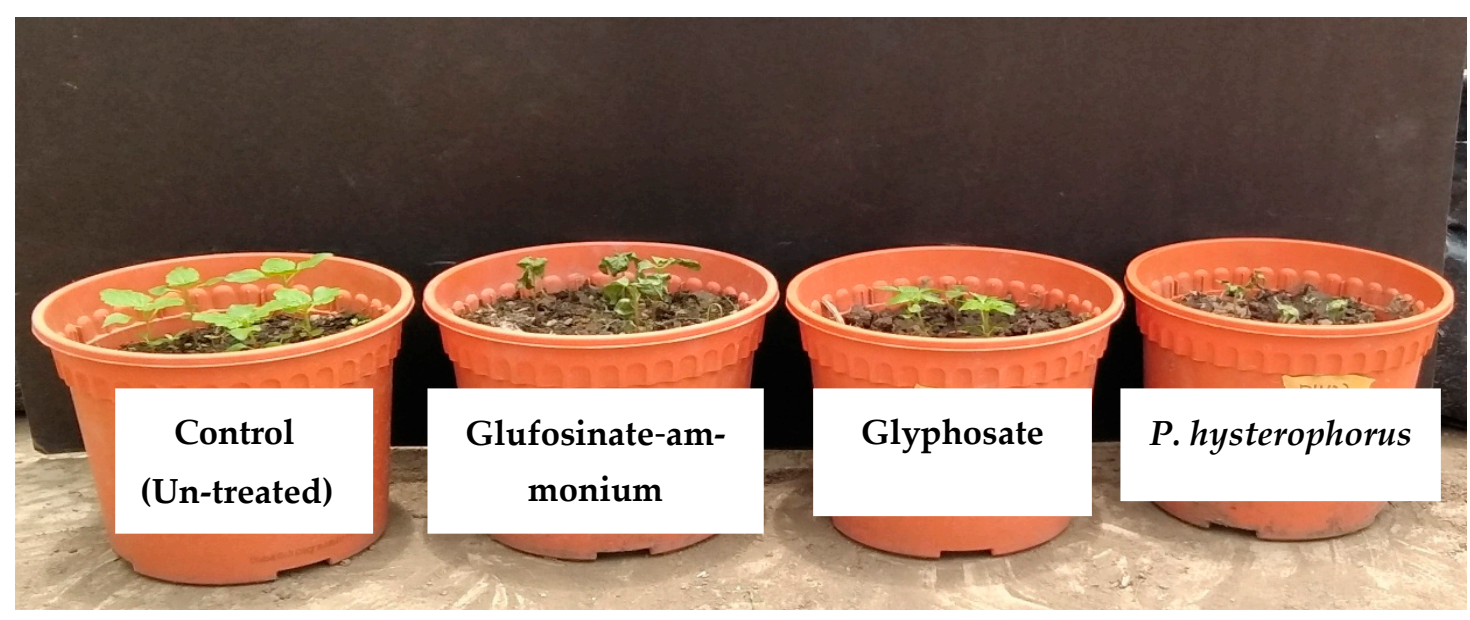

Figure 4. Effect of $P$. hysterophorus extract at $80 \mathrm{~g} \mathrm{~L}^{-1}$ concentration on A. conyzoides at $24 \mathrm{~h}$ after spray compared with glufosinate-ammonium and glyphosate herbicides.

The plant height of $A$. conyzoides, C. iria and O. sativa was inhibited by $54.32 \%, 37.71 \%$ and $26.08 \%$, respectively, when treated with $P$. hysterophorus extract at $40 \mathrm{~g} \mathrm{~L}^{-1}$. The complete inhibition of plant height of $A$. conyzoides was observed on those pots where $80 \mathrm{~g} \mathrm{~L}^{-1}$ of $P$. hysterophorus extract was sprayed, whereas $42.97 \%$ and $41.02 \%$ plant height inhibitions were observed for C. iria and O. sativa, respectively, at the same concentration. In general, there was a reduction in the fresh and dry weights of treated weeds in pots receiving $P$. hysterophorus extract. The differences in inhibitory activity among the three doses, viz. 20, 40 and $80 \mathrm{~g} \mathrm{~L}^{-1}$ of P. hysterophorus, on the fresh and dry weight of weeds, were significant. The dry weights of $A$. conyzoides, $C$. iria and $O$. sativa were inhibited by $98.63 \%, 63.80 \%$ and $62.76 \%$, respectively, when $P$. hysterophorus extract was sprayed at $80 \mathrm{~g} \mathrm{~L}^{-1}$. This result exhibited that there is no significant difference between the foliar spray of $P$. hysterophorus at $80 \mathrm{~g} \mathrm{~L}^{-1}$ and positive control when applied on $A$. conyzoides, whereas $C$. iria and $O$. sativa were less sensitive to $P$. hysterophorus extract compared to the positive control.

\section{Discussion}

The allelopathic potential of P. hysterophorus on C. iria was studied in this study. The methanol extract of $P$. hysterophorus influenced $C$. iria seedling growth and germination percentages. The extracts had a dose-dependent effect on the germination percentage, coleoptile and radicle growth of the tested weed. Plant extracts are hypothesized to impede the germination process due to the osmotic effects on the fate of imbibition, which in turn reduce the commencement of germination and, in particular, cell elongation [22]. C. iria seed germination and seedling growth were completely suppressed by $50 \mathrm{~g} \mathrm{~L}^{-1}$ of P. hysterophorus extract. Batish et al. [23], Singh et al. [24] and Mersie and Singh [25] all observed that $P$. hysterophorus extract or its residues inhibited the growth and development of several field crops. Furthermore, when compared to germination percentage and the coleoptile length, the radicle length of the test species was more sensitive to extracts. As radicles are the first organ to be exposed to phytochemicals and have more permeable tissue than other organs [21,26,27], and/or low mitotic division in the root apical meristem [28], radicle growth is more sensitive to allelopathic plant extract. Furthermore, phytochemicals can inhibit the development of radicle tissues and endoderm by affecting genes involved in cellular characterization [29].

The glasshouse experiment gave more support for the high allelopathic potential of $P$. hysterophorus extract seen in the lab. The results revealed that extracts of $P$. hysterophorus at 50 and $100 \mathrm{~g} \mathrm{~L}^{-1}$ greatly showed the growth of 21-day-old C. iria. At the mature stage of C. iria, the maximum concentration $\left(100 \mathrm{~g} \mathrm{~L}^{-1}\right)$ of P. hysterophorus extract resulted in the greatest decrease. Many researchers from all around the world have demonstrated 
dose-dependent inhibitory activity $[21,27,30,31]$. Only untreated C. iria continued flowering 21 days after spray, indicating that allelochemicals stress may have suppressed the other treated plants. Aslam et al. [32] investigated the phytotoxic effect of Calatropis procera, Peganum harmala and Tamarix aphylla on mustard and wheat shoot and root length, finding that wheat was susceptible to all three extracts at all dosages.

As the concentration of $P$. hysterophorus extract was raised, reduced dry weights and leaf area were reduced. The reduction in plant height and leaf area was discovered to be associated with a reduction in total dry weight. Several studies show that different extracts reduce the leaf area of plant species [33,34]. The dry weight of soybeans was greatly changed by the castor beans leaf aqueous extract, according to Da Silva et al. [35].

Foliar spray of $P$. hysterophorus extract reduced the Fv/Fm, photosynthesis rate, stomatal conductance and transpiration of $C$. iria. The value of Fv /Fm was significantly decreased by the foliar spray of P. hysterophorus extract. Thylakoid membrane damage and inhibition of energy transfer from antenna molecules to reaction centers can lead to photoinhibition damage and lower Fv/Fm [36]. Allelochemicals can significantly affect the performance of thylakoid electron transport during light reactions, stomatal control of carbon dioxide and the carbon cycle in dark reactions [37].

The reduction in leaf photosynthesis was attributed to a decrease in photosynthetic metabolites, carboxylation efficiency, impairment of chloroplast activity, increase in enzyme activities [38] and production of ROS caused impediment of photosynthetic mechanism [39]. Stomatal control is a vital property through which the plants limit water loss and gas exchange. These features are influenced by several determinants, including stress [40], and indicate the lower photosynthetic efficiency of plants. The carboxylation and water-use efficiency was also reduced in the plants subjected to P. hysterophorus extract.

The reduction in the transpiration rate is certainly associated with stomatal conductance. This study reveals that $P$. hysterophorus extract played a notable role in decreasing the transpiration rate for test plants at different exposure times. The concentration of phenolic acids resulted in a decline in overall water utilization and transpiration of cucumber seedlings in a linear manner [41]. The solution of cinnamic acid and benzoic acids decreased the stomatal conductance and transpiration of cucumber seedlings [42].

It was also observed in the present study that the application of plant extracts in laboratory conditions caused more inhibition compared to glasshouse as a foliar spray. Al-Humaid and El-Mergawi [43] also reported the same. The inhibition by foliar spray may occur through various mechanisms, such as a decreased rate of ion absorption, hormone and enzyme activity, cell membrane permeability and certain physiological processes, e.g., photosynthesis, respiration and protein formation [44]. Thus, the seedling and mature stage of target plants may vary in their sensitivities to plant extracts.

In this research, the methanol extract of $P$. hysterophorus was also investigated for the identification of active phytochemical constituents using LC-MS QTOF and also for their allelopathic potentiality on C. iria. Methanol was reported to be an efficient extraction solvent of lower molecular weight polyphenols [45] and a highly efficient solvent for extracting phenolic compounds compared to ethanol [46]. The results indicated the presence of phenolic compounds (flavonoids, phenols, coumarins, carboxylic acids, benzoic acids), terpenoids, alkaloids, amino acids, fatty acids, piperazines, benzofuran, indole, amines, azoles, sulfonic acid and other unknown compounds in P. hysterophorus. Among the proposed compounds, some of them have been reported as toxins in different studies. The hydroxyl group of phenolic compounds is directly attached to an aromatic ring. Phenolic allelochemicals are major allelochemicals that inhibited photosynthesis in plants [42] and modified the permeability of root cell membranes, decreased energy metabolism and inhibited cell division and root branching [47]. Research studies revealed that phenolic compounds from Chenopodium murale L. affect the growth and macromolecule content in chickpeas and peas [48].

Umbelliferone, a coumarin derivative, was found in P. hysterophorus, and, as Pan et al. [49] reported, it shows strong inhibition on lettuce and two field weeds, Setaria 
viridis and Amaranthus retroflexus. Phthalic anhydride, another compound of P. hysterophorus, formed Phthalic acid in the presence of water, which inhibited the fruit germination of Lactuca sativa L. [50]. Three terpenoids (Parthenin, Dehydroleucodine, Rishitin) and one alkaloid (9-hydroxyellipticine) were also found in P. hysterophorus extract. Many past and recent research reports revealed that terpenoids and alkaloids are also known for their allelopathic effect. Parthenin reduced the germination and growth of Avena fatua L. and Bidens pilosa L. and a dose-response relationship was observed by Batish et al. [51]. Valine is an amino acid found in P. hysterophorus, which significantly inhibited peach seedling growth [52]. Some fatty acids, amines and sulfonic acids were also observed in the LC-MS analysis of $P$. hysterophorus.

The efficacy of $P$. hysterophorus extract was increased with an increasing application rate. Similarly, the extract phytotoxicity level of Zingiber officinale increased with increasing concentration [53]. At $80 \mathrm{~g} \mathrm{~L}^{-1}, P$. hysterophorus extract produced similar efficacy to glyphosate and glufosinate on A. conyzoides. Many researchers found the efficacy of bioherbicide for weed control. For instance, Aglaia odorata leaf extract has bioherbicide properties that can hinder the growth and development of weeds [54].

Furthermore, the results also indicated that the inhibition magnitude of applied methanol extract of $P$. hysterophorus was species-dependent. The selectivity of an herbicide depends on application rate, the growth stage and morphological characteristics of the target plants and other environmental factors, which might affect the absorption, translocation and metabolism of the herbicide [55].

\section{Materials and Methods}

Graphical scheme of experimental design was presented in Figure 5.

\subsection{Test Plants}

Cyperus iria L. (Rice flatsedge) (voucher specimen\#UPMWS019), Ageratum conyzoides L. (Billygoat-weed) (voucher specimen\#UPMWS001), Oryza sativa f. spontanea Roshev (Weedy rice) (voucher specimen\#UPMWS025) were collected from the rice field of Sekinchan, Kuala Selangor, Selangor, Malaysia.

\subsection{Extraction Procedure}

The extraction was carried out conducted at Universiti Putra Malaysia's Weed Science Laboratory, which is a part of the Department of Crop Science. Methanol extracts were prepared using the method reported by Aslani et al. [56]. Parthenium hysterophorus (voucher specimen\#UPMWS0031) was obtained at its matured stage in Ladang Infoternak, Sungai Siput, Perak, Malaysia. The plants were properly washed under running tap water to remove dust particles and other debris, and then air-dried for 3 weeks in open trays under shaded conditions at room temperature $\left(25 \pm 1{ }^{\circ} \mathrm{C}\right)$. In a Willey mill, the plants were then chopped and crashed. An amount of $100 \mathrm{~g}$ powder of $P$. hysterophorus was soaked in a conical flask with $1000 \mathrm{~mL}$ methanol: distilled water $(80: 20, v / v \%)$ and the flask was wrapped in paraffin. An Orbital shaker was used to shake the flask for $48 \mathrm{~h}$ at room temperature $\left(25 \pm 1^{\circ} \mathrm{C}\right)$. The solution was filtered through four layers of cheesecloth before being centrifuged at $3000 \mathrm{rpm}$ for 1 hour. Then, a $0.2 \mathrm{~mm}$ Nalgene filter was used (Becton Dickinson Labware, Lincoln Park, NJ) to re-filter the solution. A rotary evaporator was used at $40{ }^{\circ} \mathrm{C}$ to evaporate the methanol from the extract. The mean extraction yield was $18.56 \mathrm{~g}$ from $100 \mathrm{~g}$ powdered sample of P. hysterophorus.

$$
\text { Extraction percentage }=[\text { Extract weight }(\mathrm{g}) / \text { powder weight }(\mathrm{g})] \times 100
$$

The crude sample (20 mg) was diluted into 100\% HPLC GRADE methanol $(20 \mathrm{~mL})$ and filtered with 0.2- $\mu \mathrm{m}, 15-\mathrm{mm}$ syringe filters (Phenex, Non-sterile, Luer/Slip, LT Resources, Malaysia) for LC-QTOF-MS/MS analysis. 


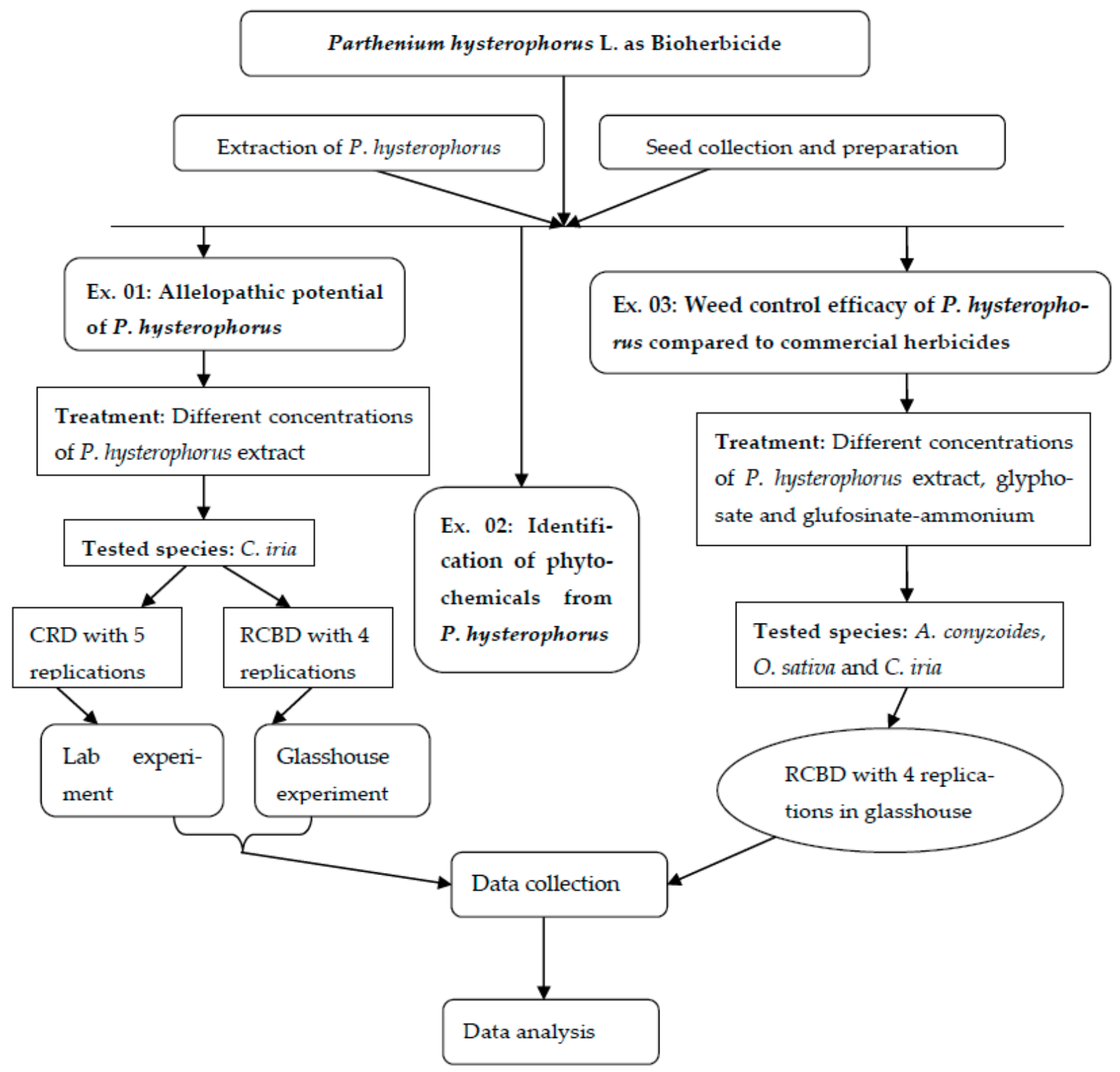

Figure 5. Graphical scheme of study design.

\subsection{Laboratory Bioassay}

From January to March 2019, the experiment was carried out in a growth chamber at the Seed Technology Laboratory, Department of Crop Science, Universiti Putra Malaysia $\left(3^{\circ} 02^{\prime} \mathrm{N}, 101^{\circ} 42^{\prime} \mathrm{E}, 31 \mathrm{~m}\right.$ elevation). Seeds were gathered that were healthy and uniform, then soaked for $24 \mathrm{~h}$ in 0.2 percent potassium nitrate $\left(\mathrm{KNO}_{3}\right)$, rinsed with distilled water and incubated at room $\left(24-26^{\circ} \mathrm{C}\right)$ temperature until the radicle emerged for about $1 \mathrm{~mm}$. Twenty uniform pre-germinated $C$. iria seeds were inserted in disposable plastic Petri dishes with a 9.0-cm-diameter and two sheets of Whatman No. 1 filter paper. After that, the filter paper on the Petri dishes was wetted and soaked with $10 \mathrm{~mL}$ of P. hysterophorus methanol extracts at six different concentrations: 0 (distilled water only), 6.25, 12.5, 25, 50 and $100 \mathrm{~g} \mathrm{~L}^{-1}$. The treatment was replicated 5 times in a completely randomized design. The Petri dishes were then incubated under fluorescent light (8500 lux) in a growth chamber at $30 / 20{ }^{\circ} \mathrm{C}$ (day/night) with a $12 \mathrm{~h} / 12 \mathrm{~h}$ (day/night cycle). The relative humidity ranged from $30 \%$ to $50 \%$. To facilitate gas exchange and avoid anaerobic conditions, the lids of the Petri dishes were not sealed. 
All seedlings germination \%, coleoptile and radicle length were assessed after 7 days. Image J software [57] was used to measure the length of the coleoptile and radicle, and the inhibitory effect was calculated using the equation below [56]:

$$
\mathrm{I}=100(\mathrm{C}-\mathrm{A}) / \mathrm{C}
$$

where "I" represents the percent inhibition, " $\mathrm{C}$ " represents the mean length of coleoptile and radicle of the control and " $\mathrm{A}$ " is the mean length of coleoptile and radicle of the methanol extracts treated seeds.

\subsection{Glasshouse Experiment}

The glasshouse experiment took place at Universiti Putra Malaysia's Faculty of Agriculture in Ladang 15 from April to June 2020. The effects of foliar application of $P$. hysterophorus methanol extracts on the growth and development of $C$. iria were investigated. Pre-germinated seeds were placed in each pot $(15 \mathrm{~cm}$ diameter $\times 12 \mathrm{~cm}$ height $)$ and covered with $1 \mathrm{~cm}$ soil, then moistened with water. Only five healthy seedlings of equal size were maintained in each pot after germination. With four replications, the pots were arranged in a randomized complete block design. Methanol extracts of $P$. hysterophorus were sprayed on examined plants (2-3 leaf stage) at doses of $6.25,12.5,25,50$ and $100 \mathrm{~g} \mathrm{~L}^{-1}$ concentrations on tested plants (2-3 leaf stage) using a $1 \mathrm{~L}$ multipurpose sprayer (Deluxe pressure sprayer). Water was used to make spray volume $\left(100 \mathrm{~mL} \mathrm{~m}^{-2}\right)$ [22]. At two-day intervals or when the soil became dry, plants in the control treatment were sprayed with $200 \mathrm{~mL}$ water without extract. Three weeks after spray, plant height, leaf area, dry weight, Fv /Fm, photosynthesis rate, transpiration and stomatal conductance were determined. Plant height was measured using $1 \mathrm{~m}$ ruler from the ground level in the pot. The leaf area was determined using leaf area meter (LI-3000, Li-COR, USA) and expressed as $\mathrm{cm}^{2}$ plant $^{-1}$. Samples were dried in an oven at $60^{\circ} \mathrm{C}$ for $72 \mathrm{~h}$; then, dry weights were determined using a digital balance. The efficiency of photosystem II in each leaf was measured with a Multi-Function Plant Efficiency Analyser (Hansatech Instruments, King's Lynn, United Kingdom). The Fv parameter (variable fluorescence) was calculated as the difference between the Fm (maximum fluorescence) and Fo (minimum fluorescence). The rate of photosynthesis, transpiration and stomatal conductance were measured from randomly selected four leaves from each test weed species using LICOR (LI-6400XT) portable photosynthesis system, (LI-COR-Inc Lincoln, Nebraska, USA) between 9:00 am to 11:00 am under bright daylight. The measurements were taken on the abaxial surface at $\mathrm{CO}_{2}$ flow rate of $400 \mu \mathrm{mol} \mathrm{m}^{-2} \mathrm{~s}^{-1}$ and the saturating photosynthetic photon flux density (PPFD) was $1000 \mathrm{mmol} \mathrm{m}^{-2} \mathrm{~s}^{-1}$ [58].

Another experiment was conducted to compare the phytotoxicity level of P. hysterophorus with synthetic herbicides. Therefore, the seeds of $A$. conyzoides, $C$. iria and $O$. sativa were seeded in the pots ( $15 \mathrm{~cm}$ diameter) and moistened with tap water. After germination, five equal-sized healthy seedlings were kept in each pot. The pots were arranged in a randomized complete block design with four replications. Methanol extracts of P. hysterophorus were sprayed with 20, 40 and $80 \mathrm{~g} \mathrm{~L}^{-1}$ concentration on tested plants (4-6 leaf stage for broadleaf and 2-3 for grasses and sedges). Plants in the negative control treatment were sprayed with $200 \mathrm{~mL}$ water without extract at 2 day intervals or when the soil became dry. Plants in the positive control treatment were sprayed with glyphosate $41 \%$ a.i. (Roundup ${ }^{\circledR}$ ) and glufosinate-ammonium $13.5 \%$ a.i. $\left(\right.$ Basta $\left.^{\circledR}\right)$ without extract $\left(2 \mathrm{~L} \mathrm{ha}^{-1} / 4.4 \mathrm{~mL} \mathrm{~L}^{-1}\right)$ at the same time when P. hysterophorus was sprayed.

Injury symptoms, plant height $(\mathrm{cm})$ and fresh and dry weights $\left(\mathrm{g} \mathrm{pot}^{-1}\right)$ were measured 3 weeks after spray. Injury symptoms were visually evaluated on test weeds using the European Weed Control and Crop Injury Evaluation scale (Table 6). 
Table 6. Injury rating scale [59].

\begin{tabular}{ccc}
\hline Scale & Injury (\%) & Effects on Weeds \\
\hline 1 & 0 & No effect (all foliage green and alive) \\
2 & $1-10$ & Very light symptoms \\
3 & $11-30$ & Light symptoms \\
4 & $31-49$ & Symptoms not reflected in yield \\
5 & 50 & Medium \\
6 & $51-70$ & Fairly heavy damage \\
7 & $71-90$ & Heavy damage \\
8 & $91-99$ & Very heavy damage \\
9 & 100 & Complete kill (dead) \\
\hline
\end{tabular}

\subsection{LC-QTOF-MS/MS Analysis}

Agilent 1290 Infinity LC system coupled to Agilent 6520 Accurate-Mass Q-TOF mass spectrometer with dual ESI source was used for analyzing chemical constituents from the methanol extract of $P$. hysterophorus. The types of the column, solvent systems and MS parameters were optimized for better analysis of the chemical profiling. ACQUITY UPLC BEH C18 column $(150 \mathrm{~mm} \times 2.1 \mathrm{~mm} \times 3.5 \mu \mathrm{m})$ was selected and held at $50{ }^{\circ} \mathrm{C}$ with a constant flow rate of $0.4 \mathrm{~mL} \mathrm{~min}^{-1}$ for providing fast and efficient separations at lower column pressures [60] and total LC run time was $26 \mathrm{~min}$. Sample elution was performed in a gradient manner using a mobile phase comprised of water (LC-MS Grade) containing $0.1 \%$ Formic acid (solvent A) and acetonitrile (LC-MS Grade) containing $0.1 \%$ Formic acid (solvent B). Nebulizer pressure was 40 psi, drying gas flow and temperature was set at $10 \mathrm{~L} \mathrm{~min}^{-1}$ and $325^{\circ} \mathrm{C}$, respectively, to perform the MS/MS experiments. In order to obtain the most sensitive ionization effect for analytes, positive and negative ion modes were investigated at different collision energy (CE) to optimize the signals and obtain maximal structure information from the ions for the mass range of 100-3200 m/z. Data processing was performed by Mass Hunter Qualitative Analysis software and peak identification was carried out based on comparison with literature values and online database [61].

\subsection{Statistical Analysis}

For all trials, a one-way analysis of variance (ANOVA) was used to see if there were any significant differences between the treatments and the control. The Tukey test with a 0.05 probability level was used to pool the differences between the treatment means. The analysis was carried out using SAS (Statistical Analysis System) software (version 9.4).

\section{Conclusions}

The current study reveals that the $P$. hysterophorus extract was capable of inhibiting the germination and growth of weeds and also confirmed the herbicidal potential compared with synthetic herbicides. The presence of 82 known compounds was also confirmed in the extract of P. hysterophorus and some of them have been reported as toxins in different studies. The great efficacy and selectivity of this weed could be characterized as a natural product to control weeds. The use of plant-based bioherbicide for weed management can increase crop yields as well as provide an alternative method of sustainable weed management. The most phytotoxic compounds from P. hysterophorus can be synthesize to develop new natural herbicides with novel modes of action. Metabolomics identification and the isolation of the major potential allelopathins, coupled with formulation techniques via multiple surfactants/nano-formulation, are also required to enhance the penetration and absorption of active compounds. 
Author Contributions: Conceptualization, A.S.J. and N.B.A.; methodology, A.S.J., N.B.A. and A.K.M.M.I.; validation, A.S.J., M.K.U. and M.M.; formal analysis, M.M., M.H. and M.S.A.-H.; investigation, M.M. and M.H.; resources, A.S.J.; data curation, A.K.M.M.I., M.M. and M.H.; writing—original draft preparation, M.M. and M.H.; writing-review and editing, A.K.M.M.I., A.S.J. and M.S.A.-H.; visualization, M.K.U., M.M. and M.H.; supervision, A.S.J., M.K.U., N.B.A., A.K.M.M.I. and M.S.A.-H.; project administration, A.S.J.; funding acquisition, A.S.J. All authors have read and agreed to the published version of the manuscript.

Funding: This research received funding from the Fundamental Research Grant Scheme (FRGS), Malaysia (FRGS/1/2013/STWN03/UPM/01/1 and FRGS/1/2017/WAB01/UPM/01/2), Putra Grant UPM (GP-IPB/2017/9523400).

Institutional Review Board Statement: Not applicable.

Informed Consent Statement: Not applicable.

Data Availability Statement: Not applicable.

Acknowledgments: The authors are grateful to the Fundamental Research Grant Scheme (FRGS), Malaysia (FRGS/1/2013/STWN03/UPM/01/1 and FRGS/1/2017/WAB01/UPM/01/2), Putra Grant UPM (GP-IPB/2017/9523400) and also sincerely acknowledge the University Putra Malaysia for providing facilities.

Conflicts of Interest: The authors declare no conflict of interest.

\section{References}

1. Holm, L.G.; Plucknett, D.L.; Pancho, J.V.; Herberger, J.P. The World's Worst Weeds: Distribution and Biology; The University Press of Hawaii: Malabar, FL, USA, 1991; p. 609.

2. Rao, A.; Johnson, D.; Sivaprasad, B.; Ladha, J.; Mortimer, A. Weed Management in Direct-Seeded Rice. Adv. Agron. 2007, 93, 153-255. [CrossRef]

3. Chauhan, B.S.; Johnson, D. Ecological studies on Cyperus difformis, Cyperus iria and Fimbristylis miliacea: Three troublesome annual sedge weeds of rice. Ann. Appl. Biol. 2009, 155, 103-112. [CrossRef]

4. Chauhan, B.S.; Johnson, D.E. Responses of Rice Flatsedge (Cyperus iria) and Barnyardgrass (Echinochloa crus-galli) to Rice Interference. Weed Sci. 2010, 58, 204-208. [CrossRef]

5. Dhammu, H.S.; Sandhu, K.S. Critical period of Cyperus iria L. competition in transplanted rice. In Proceedings of the 13th Australian Weeds Con-ference: Weeds "Threats now and forever?", Perth, Western Australia, 8-13 September 2002.

6. Shrestha, A. Weed science as a new discipline and its status in some South Asian universities and colleges: Examples from Bangladesh, Bhutan, Nepal and Pakistan. CAB Rev. 2021, 16, 1-14. [CrossRef]

7. Heap, I. Herbicide Resistant Weeds. In Integrated Pest Management; Springer Science and Business Media LLC: Berlin/Heidelberg, Germany, 2014; pp. 281-301.

8. Hussain, M.I.; Reigosa, M.J. Higher peroxidase activity, leaf nutrient contents and carbon isotope composition changes in Arabidopsis thaliana are related to rutin stress. J. Plant Physiol. 2014, 171, 1325-1333. [CrossRef] [PubMed]

9. Islam, A.K.M.M.; Yeasmin, S.; Qasem, J.R.S.; Juraimi, A.S.; Anwar, P. Allelopathy of Medicinal Plants: Current Status and Future Prospects in Weed Management. Agric. Sci. 2018, 9, 1569-1588. [CrossRef]

10. Motmainna, M.; Juraimi, A.S.; Uddin, M.K.; Asib, N.B.; Islam, A.K.M.M.; Hasan, M. Assessment of allelopathic compounds to develop new natural herbicides: A review. Allelopathy J. 2021, 52, 19-37. [CrossRef]

11. Ridenour, W.M.; Callaway, R.M. The relative importance of allelopathy in interference: The effects of an invasive weed on a native bunchgrass. Oecologia 2001, 126, 444-450. [CrossRef] [PubMed]

12. Kato-Noguchi, H. Involvement of Allelopathy in the Invasive Potential of Tithonia diversifolia. Plants 2020, 9, 766. [CrossRef]

13. Kato-Noguchi, H.; Kobayashi, A.; Ohno, O.; Kimura, F.; Fujii, Y.; Suenaga, K. Phytotoxic substances with allelopathic activity may be central to the strong invasive potential of Brachiaria brizantha. J. Plant Physiol. 2014, 171, 525-530. [CrossRef]

14. Motmainna, M.; Juraimi, A.S.B.; Uddin, K.; Asib, N.B.; Islam, A.M.; Hasan, M. Allelopathic potential of Malaysian invasive weed species to control weedy rice (Oryza sativa f. spontanea Roshev). Allelopath. J. 2021, 53, 53-68. [CrossRef]

15. Qu, T.; Du, X.; Peng, Y.; Guo, W.; Zhao, C.; Losapio, G. Invasive species allelopathy decreases plant growth and soil microbial activity. PLoS ONE 2021, 16, e0246685. [CrossRef] [PubMed]

16. Gnanavel, I.; Natarajan, S.K. Parthenium hysterophorus L.: A major threat to natural and agro eco-systems in India. Int. J. Agric. Environ. Biotech. 2013, 6, 261-269.

17. Masum, S.M.; Hasanuzzaman, M.; Ali, M.H. Threats of Parthenium hysterophorus on agro-ecosystems and its management: A review. Int. J. Agric. Crop Sci. 2013, 6, 684 .

18. Mekonnen, G. Threats and Management Options of Parthenium (Parthenium hysterophorus L.) in Ethiopia. Agric. Res. Technol. Open Access J. 2017, 10, 1-7. [CrossRef] 
19. Motmainna, M.; Juraimi, A.S.; Uddin, M.K.; Asib, N.B.; Islam, A.K.M.M.; Hasan, M. Bioherbicidal Properties of Parthenium hysterophorus, Cleome rutidosperma and Borreria alata Extracts on Selected Crop and Weed Species. Agronomy 2021, 11, 643. [CrossRef]

20. Qasem, J.R. Differences in the allelopathy results from field observations to laboratory and glasshouse experiments. Allelopath. J. 2010, 26, 45-58.

21. Islam, A.H.; Yeasmin, S.; Kader, R.A. Bioassay screening of sawdust obtained from selected tropical tree species for allelopathic properties and their field performance against paddy weeds. Fundam. Appl. Agric. 2019, 4, 906-915. [CrossRef]

22. El-Mergawi, R.A.; Al-Humaid, A.I. Searching for natural herbicides in methanol extracts of eight plant species. Bull. Natl. Res. Cent. 2019, 43, 22. [CrossRef]

23. Batish, D. Allelopathic effects of parthenin against two weedy species, Avena fatua and Bidens pilosa. Environ. Exp. Bot. 2002, 47, 149-155. [CrossRef]

24. Singh, H.P.; Batish, D.R.; Pandher, J.K.; Kohli, R. Phytotoxic effects of Parthenium hysterophorus residues on three Brassica species. Weed Biol. Manag. 2005, 5, 105-109. [CrossRef]

25. Mersie, W.; Singh, M. Allelopathic effect of parthenium (Parthenium hysterophorus L.) extract and residue on some agronomic crops and weeds. J. Chem. Ecol. 1987, 13, 1739-1747. [CrossRef] [PubMed]

26. Nishida, N.; Tamotsu, S.; Nagata, N.; Saito, C.; Sakai, A. Allelopathic Effects of Volatile Monoterpenoids Produced by Salvia leucophylla: Inhibition of Cell Proliferation and DNA Synthesis in the Root Apical Meristem of Brassica campestris Seedlings. J. Chem. Ecol. 2005, 31, 1187-1203. [CrossRef] [PubMed]

27. Islam, A.M.; Hasan, M.; Musha, M.H.; Uddin, K.; Juraimi, A.S.; Anwar, P. Exploring 55 tropical medicinal plant species available in Bangladesh for their possible allelopathic potentiality. Ann. Agric. Sci. 2018, 63, 99-107. [CrossRef]

28. Levizou, E.; Karageorgou, P.; Psaras, G.K.; Manetas, Y. Inhibitory effects of water soluble leaf leachates from Dittrichia viscosa on lettuce root growth, statocyte development and graviperception. Flora-Morphol. Distrib. Funct. Ecol. Plants 2002, 197, 152-157. [CrossRef]

29. Franco, D.M.; Silva, E.M.; Saldanha, L.L.; Adachi, S.A.; Schley, T.R.; Rodrigues, T.M.; Dokkedal, A.L.; Nogueira, F.T.S.; De Almeida, L.F.R. Flavonoids modify root growth and modulate expression of SHORT-ROOT and HD-ZIP III. J. Plant Physiol. 2015, 188, 89-95. [CrossRef]

30. Mousavi, S.S.; Karami, A.; Haghighi, T.M.; Alizadeh, S.; Maggi, F. Phytotoxic Potential and Phenolic Profile of Extracts from Scrophularia striata. Plants 2021, 10, 135. [CrossRef] [PubMed]

31. Abd-Elgawad, A.M.; El Gendy, A.E.-N.G.; Assaeed, A.M.; Al-Rowaily, S.L.; Alharthi, A.S.; Mohamed, T.A.; Nassar, M.I.; Dewir Y.H.; ElShamy, A.I. Phytotoxic Effects of Plant Essential Oils: A Systematic Review and Structure-Activity Relationship Based on Chemometric Analyses. Plants 2020, 10, 36. [CrossRef]

32. Alam, A.; Juraimi, A.S.; Rafii, M.Y.; Hamid, A.A.; Aslani, F.; Hakim, M.A. Salinity-induced changes in the morphology and major mineral nutrient composition of purslane (Portulaca oleracea L.) accessions. Biol. Res. 2016, 49, 1-19. [CrossRef]

33. Jabran, K.; Mahajan, G.; Sardana, V.; Chauhan, B.S. Allelopathy for weed control in agricultural systems. Crop. Prot. 2015, 72, 57-65. [CrossRef]

34. Algandaby, M.M.; El-Darier, S.M. Management of the noxious weed; Medicago polymorpha L. via allelopathy of some medicinal plants from Taif region, Saudi Arabia. Saudi J. Biol. Sci. 2018, 25, 1339-1347. [CrossRef] [PubMed]

35. Renathielly, F.D.S.; Rodrigo, T.B.; Bruno, M.Z.; Maurício, A.P.; Samuel, N.M.D.S.; Reginaldo, F.S.; Da Silva, R.F.; Bressan, R.T.; Zilli, B.M.; Pilatti, M.A.; et al. Allelopathic effect of aqueous extract of fresh leaf castor beans (Ricinus communis L.) applied to the beginning stage of soy (Glycine max L.) and safflower (Carthamus tinctorius L.). Afr. J. Biotechnol. 2016, 15, 2787-2793. [CrossRef]

36. Dalal, V.K.; Tripathy, B.C. Water-stress induced downsizing of light-harvesting antenna complex protects developing rice seedlings from photo-oxidative damage. Sci. Rep. 2018, 8, 1-16. [CrossRef]

37. Zhou, Y.; Yu, J. Allelochemicals and photosynthesis. In Allelopathy; Springer Science and Business Media LLC: Berlin, Germany, 2006; pp. 127-139.

38. Lawlor, D.W. Limitation to Photosynthesis in Water-stressed Leaves: Stomata vs. Metabolism and the Role of ATP. Ann. Bot. 2002, 89, 871-885. [CrossRef] [PubMed]

39. Foyer, C.H.; Shigeoka, S. Understanding Oxidative Stress and Antioxidant Functions to Enhance Photosynthesis. Plant Physiol. 2011, 155, 93-100. [CrossRef]

40. Casson, S.; Gray, J.E. Influence of environmental factors on stomatal development. New Phytol. 2008, 178, 9-23. [CrossRef] [PubMed]

41. Blum, U.; Gerig, T.M. Relationships between Phenolic Acid Concentrations, Transpiration, Water Utilization, Leaf Area Expansion, and Uptake of Phenolic Acids: Nutrient Culture Studies. J. Chem. Ecol. 2005, 31, 1907-1932. [CrossRef]

42. Li, Z.-H.; Wang, Q.; Ruan, X.; Pan, C.-D.; Jiang, D.-A. Phenolics and Plant Allelopathy. Molecules 2010, 15, 8933-8952. [CrossRef]

43. Al-Humaid, A.; El-Mergawi, R.A. Herbicidal activities of seven native plants on the germination and growth of Phalaris minor, Echinochloa crus-galli, Portulaca oleracea and Lactuca sativa. J. Agric. Sci. Technol. 2014, 4, 843-852.

44. Scognamiglio, M.; D’Abrosca, B.; Esposito, A.; Pacifico, S.; Monaco, P.; Fiorentino, A. Plant growth inhibitors: Allelopathic role or phytotoxic effects? Focus on Mediterranean biomes. Phytochem. Rev. 2013, 12, 803-830. [CrossRef] 
45. Do, Q.-D.; Angkawijaya, A.E.; Tran-Nguyen, P.L.; Huynh, L.H.; Soetaredjo, F.E.; Ismadji, S.; Ju, Y.-H. Effect of extraction solvent on total phenol content, total flavonoid content, and antioxidant activity of Limnophila aromatica. J. Food Drug Anal. 2014, 22, 296-302. [CrossRef] [PubMed]

46. Pérez, M.B.; Calderón, N.L.; Croci, C.A. Radiation-induced enhancement of antioxidant activity in extracts of rosemary (Rosmarinus officinalis L.). Food Chem. 2007, 104, 585-592. [CrossRef]

47. Marchiosi, R.; Dos Santos, W.D.; Constantin, R.P.; De Lima, R.B.; Soares, A.R.; Finger-Teixeira, A.; Mota, T.R.; De Oliveira, D.M.; Foletto-Felipe, M.D.P.; Abrahão, J.; et al. Biosynthesis and metabolic actions of simple phenolic acids in plants. Phytochem. Rev. 2020, 19, 865-906. [CrossRef]

48. Batish, D.R.; Lavanya, K.; Singh, H.P.; Kohli, R.K. Phenolic allelochemicals released by Chenopodium murale affect the growth, nodulation and macromolecule content in chickpea and pea. Plant Growth Regul. 2007, 51, 119-128. [CrossRef]

49. Pan, L.; Li, X.-Z.; Yan, Z.-Q.; Guo, H.-R.; Qin, B. Phytotoxicity of umbelliferone and its analogs: Structure-activity relationships and action mechanisms. Plant Physiol. Biochem. 2015, 97, 272-277. [CrossRef]

50. Ma, T.; Zhou, W.; Chen, L.; Wu, L.; Christie, P.; Liu, W. Toxicity of phthalate esters to lettuce (Lactuca sativa) and the soil microbial community under different soil conditions. PLoS ONE 2018, 13, e0208111. [CrossRef] [PubMed]

51. Batish, D.R.; Singh, H.P.; Pandher, J.K.; Arora, V.; Kohli, R. Phytotoxic effect of Parthenium residues on the selected soil properties and growth of chickpea and radish. Weed Biol. Manag. 2002, 2, 73-78. [CrossRef]

52. Li, S.; Peng, F.; Xiao, Y.; Gong, Q.; Bao, Z.; Li, Y.; Wu, X. Mechanisms of High Concentration Valine-Mediated Inhibition of Peach Tree Shoot Growth. Front. Plant Sci. 2020, 11, 603067. [CrossRef]

53. Han, C.-M.; Pan, K.-W.; Wu, N.; Wang, J.-C.; Li, W. Allelopathic effect of ginger on seed germination and seedling growth of soybean and chive. Sci. Hortic. 2008, 116, 330-336. [CrossRef]

54. Kato-Noguchi, H.; Suzuki, M.; Noguchi, K.; Ohno, O.; Suenaga, K.; Laosinwattana, C. A Potent Phytotoxic Substance in Aglaia odorata Lour. Chem. Biodivers. 2016, 13, 549-554. [CrossRef]

55. Blanco, F.M.G.; Ramos, Y.G.; Scarso, M.F.; Jorge, M.F.S.A.L.A.D.C. Determining the Selectivity of Herbicides and Assessing Their Effect on Plant Roots-A Case Study with Indaziflam and Glyphosate Herbicides; IntechOpen: London, UK, 2015; pp. $275-297$.

56. Aslani, F.; Juraimi, A.S.; Ahmad-Hamdani, M.S.; Omar, D.; Alam, A.; Hashemi, F.S.G.; Hakim, A.; Uddin, M.K. Allelopathic effect of methanol extracts from Tinospora tuberculata on selected crops and rice weeds. Acta Agric. Scand. Sect. B-Plant Soil Sci. 2014, 64, 165-177. [CrossRef]

57. Mirmostafaee, S.; Azizi, M.; Fujii, Y. Study of Allelopathic Interaction of Essential Oils from Medicinal and Aromatic Plants on Seed Germination and Seedling Growth of Lettuce. Agronomy 2020, 10, 163. [CrossRef]

58. Wani, A.; Hayat, S.; Ahmad, A.; Tahir, I. Efficacy of brassinosteroid analogues in the mitigation of toxic effects of salt stress in Brassica juncea plants. J. Environ. Biol. 2017, 38, 27-36. [CrossRef]

59. Burrill, L.C.; Cárdenas, J.; Locatelli, E. Field Manual for Weed Control Research; International Plant Protetion Center, Oregon State University: Corvallis, OR, USA, 1976.

60. Guiochon, G.; Gritti, F. Shell particles, trials, tribulations and triumphs. J. Chromatogr. A 2011, 1218, 1915-1938. [CrossRef]

61. Abu Bakar, F.I.; Abu Bakar, M.F.; Abdullah, N.; Endrini, S.; Fatmawati, S. Optimization of Extraction Conditions of Phytochemical Compounds and Anti-Gout Activity of Euphorbia hirta L. (Ara Tanah) Using Response Surface Methodology and Liquid Chromatography-Mass Spectrometry (LC-MS) Analysis. Evid. Based Complementary Altern. Med. 2020, 2020. [CrossRef] [PubMed] 